Pacific

Journal of

Mathematics

ENTIRE SIGN-CHANGING SOLUTIONS

WITH FINITE ENERGY TO THE

FRACTIONAL YAMABE EQUATION

Danilo Garrido AND Monica Musso

Volume $283 \quad$ No. 1

July 2016 


\title{
ENTIRE SIGN-CHANGING SOLUTIONS WITH FINITE ENERGY TO THE FRACTIONAL YAMABE EQUATION
}

\author{
DANilo GarRido AND Monica Musso
}

We show the existence of infinitely many finite energy sign-changing solutions for the fractional Yamabe-type equation

$$
(-\Delta)^{s} u=|u|^{\frac{4 s}{n-2 s}} u \quad \text { in } \mathbb{R}^{n}
$$

where $n \geq 3$ and $s \in\left(\frac{1}{2}, 1\right)$.

\section{Introduction}

We are interested in the existence of finite energy sign-changing solutions to the fractional Yamabe-type equation in $\mathbb{R}^{n}$,

$$
(-\Delta)^{s} u=\gamma|u|^{p-1} u \text { in } \mathbb{R}^{n},
$$

where $n \geq 3$ and $p$ is the fractional critical Sobolev exponent $p=(n+2 s) /(n-2 s)$. In (1), $\gamma>0$ is a constant chosen for normalization purposes as

$$
\gamma=\frac{\Gamma\left(\frac{n+2 s}{2}\right)}{\Gamma\left(\frac{n-2 s}{2}\right)} .
$$

For any $s \in(0,1),(-\Delta)^{s}$ is the nonlocal operator defined as

$$
\begin{aligned}
(-\Delta)^{s}(x) & =c(n, s) \text { P.V. } \int_{\mathbb{R}^{n}} \frac{u(x)-u(y)}{|x-y|^{n+2 s}} d y \\
& =c(n, s) \lim _{\epsilon \rightarrow 0^{+}} \int_{\mathbb{R}^{n} \backslash B(x, \epsilon)} \frac{u(x)-u(y)}{|x-y|^{n+2 s}} d y,
\end{aligned}
$$

where P.V. stands for the principal value and

$$
c(n, s)=\pi^{-\left(2 s+\frac{n}{2}\right)} \frac{\Gamma\left(\frac{n}{2}+s\right)}{\Gamma(-s)} .
$$

This nonlocal operator in $\mathbb{R}^{n}$ can be expressed as a generalized Dirichlet-to-Neumann map for a certain elliptic boundary value problem with local differential operators

MSC2010: 35J60, 35J61, 35J75, 35R11.

Keywords: fractional Laplacian, energy solutions, Yamabe-type equation. 
defined on the upper halfspace $\mathbb{R}_{+}^{n+1}=\left\{(x, t): x \in \mathbb{R}^{n}, t>0\right\}$, as we learn from Caffarelli and Silvestre [2007]: given a solution $u=u(x)$ of $(-\Delta)^{s} u=f$ in $\mathbb{R}^{n}$, one can equivalently consider the dimensionally extended problem for $u=u(x, t)$ which solves

$$
\left\{\begin{array}{cl}
\operatorname{div}\left(t^{1-2 s} \nabla u\right)=0, & \text { in } \mathbb{R}_{+}^{n+1}, \\
-\lim _{t \rightarrow 0} d_{s} t^{1-2 s} \partial_{t} u(x, t)=f, & \text { on } \partial \mathbb{R}_{+}^{n+1},
\end{array}\right.
$$

where $d_{s}$ is the positive constant $d_{s}=2^{2 s-1} \Gamma(s) / \Gamma(1-s)$. By finite energy solutions of (1), we mean the following. Consider the Schwartz space $\mathcal{S}$ of rapidly decaying $C^{\infty}$ functions on $\mathbb{R}^{n}$, and for any $\tau \in \mathcal{S}$ we denote by

$$
\mathcal{F} \tau(\xi)=\frac{1}{(2 \pi)^{n / 2}} \int_{\mathbb{R}^{n}} e^{-i \xi \cdot x} \tau(x) d x
$$

the Fourier transformation of $\tau$. We look for solutions $u$ of (1) in the energy space

$$
\mathcal{D}^{s}\left(\mathbb{R}^{n}\right)=\left\{u \in L^{\frac{2 n}{n-2 s}}\left(\mathbb{R}^{n}\right):\left\|(-\Delta)^{\frac{s}{2}} u\right\|_{L^{2}\left(\mathbb{R}^{n}\right)}<\infty\right\},
$$

where $\left\|(-\Delta)^{\frac{s}{2}} u\right\|_{L^{2}\left(\mathbb{R}^{n}\right)}$ is defined by $\left(\int_{\mathbb{R}^{n}}|\xi|^{2 s}|\mathcal{F} u(\xi)|^{2} d \xi\right)^{\frac{1}{2}}$, endowed with the norm $\|u\|_{\mathcal{D}^{s}\left(\mathbb{R}^{n}\right)}=\left\|(-\Delta)^{\frac{s}{2}} u\right\|_{L^{2}\left(\mathbb{R}^{n}\right)}$. These solutions correspond to critical points of the functional

$$
J(u)=\frac{1}{2} \int_{\mathbb{R}^{n}}\left|(-\Delta)^{\frac{s}{2}} u\right|^{2}-\gamma \frac{n-2 s}{2 n} \int_{\mathbb{R}^{n}}|u|^{\frac{2 n}{n+2 s}}, \quad u \in \mathcal{D}^{s}\left(\mathbb{R}^{n}\right) .
$$

Following the work by Lieb [1983] — see also [Frank and Lieb 2010; 2012; Carlen and Loss 1990] for alternative proofs - positive solutions to (1) are given by the family of functions defined by

$$
U(x)=\left(\frac{2}{1+|x|^{2}}\right)^{\frac{n-2 s}{2}} \text { and } \mu^{-\frac{n-2 s}{2}} U\left(\frac{x-\xi}{\mu}\right)
$$

for any $\mu>0$ and $\xi \in \mathbb{R}^{n}$. Indeed these functions realize the Hardy-LittlewoodSobolev inequality, which states the existence of a positive number $S$ such that for all $u \in C^{\infty}\left(\mathbb{R}^{n}\right)$,

$$
S\|u\|_{L^{2^{*}\left(\mathbb{R}^{n}\right)}} \leq\left\|(-\Delta)^{\frac{s}{2}} u\right\|_{L^{2}\left(\mathbb{R}^{n}\right)}
$$

where $2^{*}=p+1=2 n /(n-2 s)$. Indeed, these functions are the only positive solutions to (1) under some decay conditions [Chen et al. 2006; Li 2004; Li and Zhu 1995]. In particular, this is true if $u \in L_{\text {loc }}^{2 n /(n-2 s)}\left(\mathbb{R}^{n}\right)$, as shown in [Chen et al. 2006].

On the other hand, (1) can be read on the sphere $S^{n} \subset \mathbb{R}^{n+1}$, after a stereographic projection. Indeed, the inverse of the stereographic projection $\pi: \mathbb{R}^{n} \rightarrow S^{n} \backslash\{S\}$, 
where $S=(0, \ldots, 0,-1) \in \mathbb{R}^{n+1}$, defined by

$$
\pi(y)=\left(\frac{2 y}{1+|y|^{2}}, \frac{1-|y|^{2}}{1+|y|^{2}}\right)
$$

is a conformal map and $\pi^{*} g_{0}=U^{\frac{4 s}{n-2 s}}(y) d y$, where $g_{0}$ is the standard metric on $S^{n}$ and $U$ is defined in (3). In $S^{n}$, the fractional Laplacian $(-\Delta)^{s}$ reduces to an elliptic pseudodifferential operator $P_{s}^{g_{0}}$ of order $2 s$ with principal symbol $\sigma_{2 s}\left(P_{s}^{g_{0}}\right)=|\xi|_{g_{0}}^{2} s$. In [Chang and González 2011] a relation between this operator and a Dirichlet-to-Neumann operator of uniformly nondegenerate elliptic boundary value problems in the spirit of [Caffarelli and Silvestre 2007] is established. We have $\pi^{*}\left(L_{s}^{g_{0}} v\right)=U^{-(n+2 s) /(n-2 s)}(-\Delta)^{s}\left(U \pi^{*} v\right)$ for any $v$ defined on $S^{n}$. Thus $u$ is a solution to (1) if and only if $w$, defined by $u=U \pi^{*} w$, solves

$$
\Delta_{g_{0}} w+\gamma\left(|w|^{\frac{4 s}{n-2 s}} w-w\right)=0 \quad \text { in } S^{n} .
$$

Positive solutions to (4) solve the so-called fractional Yamabe problem on the sphere $S^{n}$. We refer to [González and Qing 2013] for a general formulation of the fractional Yamabe problem and results concerning its solvability.

Finite energy sign-changing solutions to (1), or equivalently (4), are poorly understood.

The purpose of this paper is to give a first example of finite energy sign-changing solutions to (1), in all dimensions $n \geq 3$, and for $s \in\left(\frac{1}{2}, 1\right)$ : we build a solution to (1) which looks like the solution $U$ surrounded by $k$ negative copies $U$ properly scaled and distributed along the vertices of a regular polygon with radius 1 . Our main result is the following theorem:

Theorem 1.1. Let $n \geq 3$ and $s \in\left(\frac{1}{2}, 1\right)$. Write $\mathbb{R}^{n}=\mathbb{C} \times \mathbb{R}^{n-2}$ and let $\xi_{j}^{k}=$ $\left(e^{2 j \pi i / k}, 0\right), j=1, \ldots, k$. Then for any sufficiently large $k$, there is a finite energy solution to Problem (1) of the form

$$
u_{k}(x)=U(x)-\sum_{j=1}^{k} \mu_{k}^{-\frac{n-2 s}{2}} U\left(\mu_{k}^{-1}\left(x-\xi_{j}\right)\right)+o(1),
$$

where

$$
\mu_{k}=\left(k^{2} 2^{\frac{n-2 s}{2}} \sum_{j=1}^{\infty} j^{2 s-n}\right)^{-1}(1+o(1))
$$

Moreover,

$$
J\left(u_{k}\right)=(k+1) J(U)+O(1) .
$$

Here $O(1)$ remains bounded and $o(1) \rightarrow 0$ uniformly as $k \rightarrow+\infty$.

The proof of the result consists in defining a first approximation and then showing that a small perturbation of this approximation provides an actual solution to the 
problem. This is done by linearizing the equation around the approximation and applying an invertibility theory for the linearized operator. In this step, we use the nondegeneracy property of $U$ proved in [Dávila et al. 2013], which states that all bounded solutions of the linear problem

$$
(-\Delta)^{s} \phi-\gamma \frac{n+2 s}{n-2 s} U^{\frac{4 s}{n-2 s}} \phi=0
$$

are linear combinations of

$$
\partial_{x_{j}} U(x), \quad \text { for } j=1, \ldots, n,
$$

and

$$
\frac{n-2 s}{2} U(x)+x \cdot \nabla U(x) .
$$

Indeed, the above functions belong to the kernel of the linearized operator, due to the corresponding rigid motion under which (1) is invariant. These are the only nontrivial elements of the kernel according to [Dávila et al. 2013].

A second ingredient we take advantage of to produce an invertibility theory is the symmetry of the configuration. This reflects into the fact that our approximation, as well as our final solution, satisfy the symmetries

$$
\begin{aligned}
u\left(\bar{y}, y^{\prime}\right) & =u\left(e^{\frac{2 \pi j}{k} l} \bar{y}, y^{\prime}\right), & & j=1, \ldots, k-1, \\
u\left(y_{1}, y_{2}, \ldots, y_{j}, \ldots, y_{n}\right) & =u\left(y_{1}, y_{2}, \ldots,-y_{j}, \ldots, y_{n}\right) & & j=2, \ldots, n .
\end{aligned}
$$

Furthermore, they are invariant under Kelvin transform, namely

$$
u(y)=|y|^{2 s-n} u\left(\frac{y}{|y|^{2}}\right) .
$$

The final step in the proof consists in adjusting properly the parameter $\mu_{k}$. A detailed description of the scheme of the proof is given in Section 2.

Let us mention that a very similar construction for finite energy, sign-changing solutions to the classic Yamabe-type problem in $\mathbb{R}^{n}$ :

$$
\Delta u+|u|^{\frac{4}{n-2}} u=0 \quad \text { in } \mathbb{R}^{n},
$$

namely when $s=1$ in (1), has been done in [del Pino et al. 2011; 2013]. Indeed, our result extends to the case $s \in\left(\frac{1}{2}, 1\right)$, the construction done in [del Pino et al. 2011], from which we are inspired.

We learned recently of [Fang 2014], where the author constructs solutions to (1) similar to ours, covering the whole range $s \in(0,1)$. Nevertheless, in that case, the concentration parameter $\mu_{k}$ is of order $k^{-3}$ [Fang 2014, (2.4)], while our concentration parameter is $\mu_{k} \sim k^{-2}$, as $k \rightarrow \infty$. It is not clear to us how this choice of the parameter's rate provides a real solution to (1). Indeed, it is this choice of 
the parameter's rate, in terms of $k$, that allows the author of [Fang 2014] to cover the whole range $s \in(0,1)$.

Our restriction on $s$ is consequence of two inequalities: we need a certain power of integrability $q$ to be $q<n$ in order to have a good first approximation when estimated in proper norms, and at the same time we need $q>n /(2 s)$ to guarantee enough regularity. These constraints restrict us to $s \in\left(\frac{1}{2}, 1\right)$. We believe that our construction should work in the whole range $s \in(0,1)$, and in fact we think that $\mu_{k} \sim k^{-2}$, as $k \rightarrow \infty$, for the whole range $s \in(0,1)$, but an invertibility theory on different weighted Sobolev spaces is needed. We will treat this problem in a forthcoming paper.

The rest of the paper will be devoted to the proof of Theorem 1.1.

\section{Ansatz for the solution and scheme of the proof}

This section is devoted to define a first approximation for a solution to (1) and to describe the scheme of the proof of our result.

We start reminding that $U$ defined in (3) is invariant under Kelvin transform, namely

$$
U(y):=|y|^{2 s-n} U\left(|y|^{-2} y\right) .
$$

Even more, it can be proved that also the family of solutions

$$
\mu^{-\frac{n-2 s}{2}}\left(\frac{y-\xi}{\mu}\right)
$$

is invariant under Kelvin transform if and only if

$$
|\xi|^{2}+\mu^{2}=1 \text {. }
$$

Let $k$ be a positive integer and define, for any $j=1, \ldots, k$, the $k$ points

$$
\xi_{j}=\sqrt{1-\mu^{2}}\left(e^{2 \pi i(j-1) / k}, 0, \ldots, 0\right) \in \mathbb{R}^{2} \times \mathbb{R}^{n-2},
$$

where $\mu>0$ is a positive number of the form

$$
\mu=\frac{\delta}{k^{2}}, \quad \text { with } c<\delta<c^{-1}
$$

for a certain constant $c>0$, independent of $k$, as $k \rightarrow \infty$. Define

$$
U_{*}(y)=U(y)-\sum_{j=1}^{k} U_{j}(y), \quad \text { where } U_{j}(y)=\mu^{-\frac{n-2 s}{2}} U\left(\mu^{-1}\left(y-\xi_{j}\right)\right) .
$$

For large values of $k$, which at the same time make the scaling parameters $\mu$ very small, we shall show that $U_{*}$ is a good approximate solution for (1). Observe that 
the function $U_{*}$ satisfies the symmetry properties (6) and (7). Furthermore, $U_{*}$ is invariant under Kelvin transform

$$
U_{*}(y)=|y|^{2-n} U_{*}\left(\frac{y}{|y|^{2}}\right) .
$$

This is consequence of a straightforward computation, using the fact that

$$
\mu^{2}+\left|\xi_{j}\right|^{2}=1 \text { for any } j=1, \ldots, k \text {. }
$$

We will show that (1) admits a solution of the form

$$
u(y)=U_{*}(y)+\phi(y)
$$

where $\phi$ is small when compared with $U_{*}$. It satisfies the symmetry conditions (6) and (7), and it is invariant under Kelvin transform. Then (1) can be rewritten in terms of $\phi$ as

$$
(-\Delta)^{s} \phi-p \gamma\left|U_{*}\right|^{p-1} \phi-E-\gamma N(\phi)=0,
$$

where $E$ is

$$
\gamma^{-1} E=\left|U-\sum U_{j}\right|^{p-1}\left(U-\sum U_{j}\right)-\left(U^{p}-\sum U_{j}^{p}\right)
$$

and

$$
N(\phi)=\left|U_{*}+\phi\right|^{p-1}\left(U_{*}+\phi\right)-\left|U_{*}\right|^{p-1}-\left|U_{*}\right|^{p-1} U_{*}-p\left|U_{*}\right|^{p-1} \phi .
$$

The size of the error term $E$ defined in (11) turns out to be relatively small, as the number $k$ tends to infinity, when estimated with proper norms. Let us fix a number $q>\frac{n}{2 s}$; we define the weighted $L^{q}$ norm

$$
\|h\|_{* *}:=\left\|(1+|y|)^{n+2 s-2 n / q} h\right\|_{L^{q}\left(\mathbb{R}^{n}\right)}
$$

Let $\eta>0$ be a small and fixed number, independent of $k$. The error can be estimated separately in the exterior region $\bigcap_{j}\left\{\left|y-\xi_{j}\right|>\frac{\eta}{k}\right\}$ and then in each of the inner regions $\left\{\left|y-\xi_{j}\right|<\frac{\eta}{k}\right\}$. Indeed, we shall prove that there exists a constant $C$ such that, for all $k$ large enough,

$$
\left\|(1+|y|)^{n+2 s-2 n / q} E\right\|_{L^{q}\left(\bigcap_{j}\left\{\left|y-\xi_{j}\right|>\frac{\eta}{k}\right\}\right)} \leq C k^{1-n / q} .
$$

Observe that, in order to have a small (in $k$ ) size for the error in the exterior domain, we need $q<n$. On the other hand, for regularity issue we will discuss later, we assume that $q>\frac{n}{2 s}$. The set of possible values for $q, \frac{n}{2 s}<q<n$, is not empty since we are considering $s$ in the range $s \in\left(\frac{1}{2}, 1\right)$. 
If we change scale $\tilde{E}_{j}(y):=\mu^{\frac{n+2 s}{2}} E\left(\xi_{j}+\mu y\right)$, in $|y|<\eta /(\mu k)$, for any $j=$ $1, \ldots, k$, we have the following estimate for the error in each interior domain:

$$
\left\|(1+|y|)^{n+2 s-2 n / q} \tilde{E}_{j}(y)\right\|_{L^{q}\left\{|y|<\frac{\eta}{k \mu}\right\}} \leq C k^{-n / q} .
$$

We shall prove the validity of estimates (13) and (14) at the end of this section.

In order to solve in $\phi$ the nonlinear Equation (10), we use a gluing method. Let $\zeta$ be a cutoff function defined as follows: $\zeta(t)=1$ for $t<1$ and $\zeta(t)=0$ for $t>2$. We also defined $\zeta^{-}(t)=\zeta(2 t)$. Then we set

Observe that

$$
\zeta_{j}(y)= \begin{cases}\zeta\left(k \eta^{-1}|y|^{-2}\left|y-\xi_{j}\right| y||\right) & \text { if }|y|>1, \\ \zeta\left(k \eta^{-1}\left|y-\xi_{j}\right|\right) & \text { if }|y| \leq 1 .\end{cases}
$$

$$
\zeta_{j}(y)=\zeta_{j}\left(|y|^{-2} y\right)
$$

A function $\phi$ of the form

$$
\phi=\sum_{j=1}^{k} \tilde{\phi}_{j}+\psi
$$

is a solution of the problem (10), provided that we can solve the following coupled system of elliptic equation in $\left(\tilde{\phi}_{1}, \tilde{\phi}_{2}, \ldots, \tilde{\phi}_{k}\right)$ and $\psi$ :

$$
\begin{aligned}
(-\Delta)^{s}\left(\tilde{\phi}_{j}\right)-p \gamma\left|U_{*}\right| \zeta_{j} \tilde{\phi}_{j} & \\
& -\zeta_{j}\left(p \gamma\left|U_{*}\right|^{p-1} \psi+E+\gamma N\left(\tilde{\phi}_{j}+\sum_{i \neq j} \tilde{\phi}_{i}+\psi\right)\right)=0,
\end{aligned}
$$

where $j=1,2, \ldots, k$ and

$$
\begin{aligned}
& (-\Delta)^{s} \psi-p \gamma U^{p-1} \psi \\
& -\left(p \gamma\left(\left|U_{*}\right|^{p-1}-U^{p-1}\right)\left(1-\sum_{j=1}^{k} \zeta_{j}\right)+p \gamma U^{p-1} \sum_{j=1}^{k} \zeta_{j}\right) \psi \\
& -p \gamma\left|U_{*}\right|^{p-1} \sum_{j}\left(1-\zeta_{j}\right) \tilde{\phi}_{j} \\
& -\left(1-\sum_{j=1}^{k} \zeta_{j}\right)\left(E+\gamma N\left(\sum_{j=1}^{k} \tilde{\phi}_{j}+\psi\right)\right)=0
\end{aligned}
$$

To solve the above coupled system, we follow the following strategy. First we solve (17) in the unknown $\psi$, assuming that $\tilde{\phi}_{j}$ are fixed functions satisfying

$$
\begin{aligned}
\tilde{\phi}_{j}\left(\bar{y}, y^{\prime}\right) & =\tilde{\phi}_{1}\left(e^{\frac{2 \pi j}{k} l} \bar{y}, y^{\prime}\right), \quad j=1,2, \ldots, k-1 \\
\tilde{\phi}_{1}\left(y_{1}, y_{2}, \ldots, y_{j}, \ldots, y_{n}\right) & =\tilde{\phi}_{1}\left(y_{1}, y_{2}, \ldots,-y_{j}, \ldots, y_{n}\right) \quad j=2, \ldots, n
\end{aligned}
$$


and the invariant condition under Kelvin's transform,

$$
\tilde{\phi}_{1}=|y|^{2 s-n} \tilde{\phi}_{1}\left(|y|^{-2} y\right) \text {. }
$$

Furthermore, we assume that

$$
\left\|\phi_{1}\right\|_{*}<\rho \quad \text { where } \phi_{1}=\mu^{\frac{n-2 s}{2}} \tilde{\phi}_{1}\left(\xi_{1}+\mu y\right) .
$$

We have the validity of the following result:

Proposition 2.1. There exist constants $k_{0}, C, \rho_{0}$ such that for all $k \geq k_{0}$, the following holds: Suppose that $\tilde{\phi}_{j}, j=1,2, \ldots, k$, satisfy conditions (18)-(21) with $\rho<\rho_{0}$. Then there exists a unique solution $\psi=\Psi\left(\phi_{1}\right)$ to (17) that satisfies the symmetries

$$
\begin{array}{rlrl}
\psi\left(\bar{y}, y^{\prime}\right) & =\psi\left(e^{\frac{2 \pi j}{k} l} \bar{y}, y^{\prime}\right), & j=1,2, \ldots, k-1, \\
\psi\left(\bar{y}, \ldots, y_{j}, \ldots, y_{n}\right) & =\psi\left(\bar{y}, \ldots,-y_{j}, \ldots, y_{n}\right), & \\
\psi & =|y|^{2 s-n} \psi\left(|y|^{-2} y\right), & \\
\|\psi\|_{*} & \leq \frac{C}{k^{n / q-1}}+C\left\|\phi_{1}\right\|_{*}^{2} .
\end{array}
$$

Moreover, the operator $\Psi$ satisfies the Lipschitz condition

$$
\left\|\Psi\left(\phi_{1}^{1}\right)-\Psi\left(\phi_{1}^{2}\right)\right\|_{*} \leq C\left\|\phi_{1}^{1}-\phi_{1}^{2}\right\|_{*} .
$$

Once we have the result of the above Proposition, under the assumption on $\tilde{\phi}_{j}$ we have that all equations (16) reduce to just one, say that for $\tilde{\phi}_{1}$. Then we will find a solution to our problem if we solve

$$
(-\Delta)^{s} \tilde{\phi}_{1}-p \gamma\left|U_{1}\right|^{p-1} \tilde{\phi}_{1}-\zeta_{1} E-\gamma \mathcal{N}\left(\phi_{1}\right)=0 \quad \text { in } \mathbb{R}^{n}
$$

where

$$
\begin{aligned}
\mathcal{N}\left(\phi_{1}\right)=p\left(\left|U_{*}\right|^{p-1} \zeta_{1}-\left|U_{1}\right|^{p-1}\right) & \phi_{1} \\
& +\zeta_{1}\left(p\left|U_{*}\right|^{p-1} \Psi\left(\phi_{1}\right)+N\left(\tilde{\phi}_{1}+\sum_{i \neq 1} \tilde{\phi}_{i}+\Psi\left(\phi_{1}\right)\right)\right)
\end{aligned}
$$

Rather than solving (22) directly, we shall first solve the corresponding projected version of (22):

$$
(-\Delta)^{s} \tilde{\phi}_{1}-p \gamma\left|U_{1}\right|^{p-1} \tilde{\phi}_{1}-\zeta_{1} E+\gamma \mathcal{N}(\phi)=c_{n+1} U_{1}^{p-1} \tilde{Z}_{n+1} \quad \text { in } \mathbb{R}^{n}
$$

where

$$
c_{n+1}=-\frac{\int_{\mathbb{R}^{n}}\left(\zeta_{1} E+\gamma \mathcal{N}(\phi)\right) \tilde{Z}_{n+1}}{\int_{\mathbb{R}^{n}} U_{1}^{p-1} \tilde{Z}_{n+1}^{2}} .
$$


and

$$
\tilde{Z}_{n+1}(y)=\mu^{-\frac{n-2 s}{2}} Z_{n+1}\left(\mu^{-1}\left(y-\xi_{1}\right)\right)
$$

Proposition 2.2. There exist constants $k_{0}, C$ such that for all $k \geq k_{0}$, the following holds: Let $\Psi\left(\phi_{1}\right)$ the solution predicted by Proposition 2.1. Then there exists a unique solution $\psi_{1}=\Phi(\delta), c_{n+1}=c_{n+1}(\delta)$ to (23) and (24), which depends continuously on $\delta$. Moreover,

$$
\|\Phi\|_{*} \leq C k^{-\frac{n}{q}} \quad \text { and } \quad\|\mathcal{N}(\phi)\|_{* *} \leq C k^{-\frac{2 n}{q}}
$$

for some fixed positive constant $C$.

To conclude our argument, we shall show the existence of a number $\delta$ in the definition of $\mu$ in (8) so that the above constant $c_{n+1}$ is equal to zero. In this way, we constructed a solution to (1) with the qualitative properties predicted by Theorem 1.1.

Scheme of the paper. In Section 3 we prove some basic results on linear problems in $\mathbb{R}^{n}$. These results will be applied to prove Propositions 2.1 and 2.2 in Section 4. Section 5 is dedicated to show the existence of $\delta>0$ so that $c_{n+1}=0$, concluding in this way the proof of our theorem.

We finish this section with the proof of estimates (13) and (14).

Proof of (13). This is in the region $\bigcap_{j}\left\{\left|y-\xi_{j}\right|>\frac{\eta}{k}\right\}$. For any $y$ in this exterior region,

$$
|E(y)| \leq C\left(\frac{1}{\left(1+|y|^{2}\right)^{2 s}}+\left|\sum_{j=1}^{k} \frac{\mu^{\frac{n-2 s}{2}}}{\left|y-\xi_{j}\right|^{n-2 s}}\right|^{\frac{4 s}{n-2 s}}\right)\left(\sum_{j=1}^{k} \frac{\mu^{\frac{n-2 s}{2}}}{\left|y-\xi_{j}\right|^{n-2 s}}\right),
$$

for some positive constant $C>0$. Since for any $j$ fixed and $\left|y-\xi_{j}\right|=\frac{\eta}{k}$ we have

$$
\sum_{i=1}^{k} \frac{\mu^{\frac{n-2 s}{2}}}{\left|y-\xi_{i}\right|^{n-2 s}}=\frac{1}{k^{n-2 s}} k^{n-2 s}+\sum_{i \neq j}^{k} \frac{\mu^{\frac{n-2 s}{2}}}{\left|y-\xi_{i}\right|^{n-2 s}} \leq 1+\frac{k-1}{c k^{n-2 s}},
$$

then we conclude that

$$
|E| \leq C \frac{\mu^{\frac{n-2 s}{2}}}{\left(1+|y|^{2}\right)^{2 s}} \sum_{j=1}^{k} \frac{1}{\left|y-\xi_{j}\right|^{n-2 s}} .
$$


Thus a direct computation gives

$$
\begin{aligned}
\left\|(1+|y|)^{n+2 s-2 n / q} E\right\|_{L^{q}(\mathrm{Ext})} & \\
& \leq C \mu^{\frac{n-2 s}{2}}\left\|\frac{\left.(1+|y|)^{n+2 s-2 n / q}\right)}{\left(1+|y|^{2}\right)^{2 s}} \sum_{j=1}^{k} \frac{1}{\left|y-\xi_{j}\right|^{n-2 s}}\right\|_{L^{q}(\mathrm{Ext})} \\
& \leq C \mu^{\frac{n-2 s}{2}} \sum_{j=1}^{k}\left(\int_{\left|y-\xi_{j}\right|>\frac{\eta}{k}} \frac{(1+|y|)^{(n+2 s) q-2 n}}{\left(1+|y|^{2}\right)^{2 s q}} \frac{1}{\left|y-\xi_{j}\right|^{(n-2 s) q}} d y\right)^{\frac{1}{q}} \\
& \leq C \mu^{\frac{n-2 s}{2}} k\left(\int_{\frac{\eta}{k}}^{1} \frac{t^{n-1}}{t^{(n-2 s) q}} d t\right)^{\frac{1}{q}} \\
& =C \mu^{\frac{n-2 s}{2}} k\left(k^{(n-2 s) q-n}-1\right)^{\frac{1}{q}} \\
& \leq C \mu^{\frac{n-2 s}{2}} k^{(n-2 s)+1-\frac{n}{q}}
\end{aligned}
$$

Proof of (14). This is in the inner region $\left|y-\xi_{j}\right|<\frac{\eta}{k}$, for some $j$ fixed. Observe that if $y$ is close to $\xi_{j}$, then

$$
U_{j} \sim O\left(\mu^{-(n-2 s) / 2}\right)
$$

For any $y$ in this region, there exists $t \in(0,1)$ such that

$$
E=p\left(-U_{j}+t\left(-\sum_{i \neq j} U_{j}+U\right)\right)^{p-1}\left(-\sum_{i \neq j} U_{j}+U\right)-U^{p}+\sum_{i \neq j} U_{j} .
$$

We consider the change of scale $\tilde{E}_{j}(y):=\mu^{\frac{n+2 s}{2}} E\left(\xi_{j}+\mu y\right),|y|<\frac{\eta}{\mu k}$. Therefore, we obtain that for some $t \in(0,1)$

$$
\begin{aligned}
\tilde{E}_{j}(y)=p\left(-U(y)+t\left(\sum_{i \neq j} U\left(y-\mu^{-1}\left(\xi_{i}-\xi_{j}\right)\right)+\mu^{\frac{n-2 s}{2}} U\left(\xi_{j}+\mu y\right)\right)\right)^{p-1} \\
\times\left(-\sum_{i \neq j} U\left(y-\mu^{-1}\left(\xi_{i}-\xi_{j}\right)\right)+\mu^{\frac{n-2 s}{2}} U\left(\xi_{j}+\mu y\right)\right) \\
+\sum_{i \neq j} U^{p}\left(y-\mu^{-1}\left(\xi_{i}-\xi_{j}\right)\right)-\mu^{\frac{n+2 s}{2}} U^{p}\left(\xi_{j}+\mu y\right) .
\end{aligned}
$$

Taking into account the configuration of the points $\xi_{j}$, we have

$$
\left|\xi_{i}-\xi_{j}\right| \sim \frac{|i-j|}{k} .
$$


Furthermore, for $i \neq j$ and $|y|<\frac{\eta}{k \mu}$,

$$
\begin{aligned}
U\left(y-\mu^{-1}\left(\xi_{i}-\xi_{j}\right)\right) & \leq C \frac{\mu^{n-2 s}}{\left|\xi_{j}-\xi_{i}\right|^{n-2 s}}\left(\frac{\left|\xi_{j}-\xi_{i}\right|^{2}}{\mu^{2}+\left|\mu y-\left(\xi_{j}-\xi_{i}\right)\right|^{2}}\right)^{\frac{n-2 s}{2}} \\
& \leq C \frac{\mu^{n-2 s} k^{n-2 s}}{|i-j|^{n-2 s}} .
\end{aligned}
$$

Moreover,

$$
\mid \sum_{i \neq j} U\left(y-\mu^{-1}\left(\xi_{i}-\xi_{j}\right) \mid \leq C k^{n-2 s} \mu^{n-2 s} \quad \text { and } \quad \mu^{\frac{n-2 s}{2}} U\left(\xi_{j}+\mu y\right)\right) \leq C \mu^{\frac{n-2 s}{2}}
$$

for some constant $C>0$. Thus we conclude that

$$
\left|\tilde{E}_{j}(y)\right| \leq C\left(\frac{k^{n-2 s} \mu^{n-2 s}}{1+|y|^{4 s}}+\mu^{\frac{n+2 s}{2}}\right),
$$

and we have an estimate of the error in the inner region

$$
\begin{aligned}
& \left\|(1+|y|)^{n+2 s-\frac{2 n}{q}} \tilde{E}_{j}(y)\right\|_{L^{q}\left\{|y|<\frac{\eta}{k \mu}\right\}} \\
& \quad \leq C\left\|(1+|y|)^{n+2 s-\frac{2 n}{q}}\left(\frac{k^{n-2 s} \mu^{n-2 s}}{1+|y|^{4 s}}+\mu^{\frac{n+2 s}{2}}\right)\right\|_{L^{q}\left\{|y|<\frac{\eta}{k \mu}\right\}} .
\end{aligned}
$$

Since

$$
\begin{aligned}
\left\|(1+|y|)^{n-2 s-\frac{2 n}{q}}\right\|_{L^{q}\left\{|y|<\frac{\eta}{k \mu}\right\}}^{q} & \leq C \int_{0}^{\frac{\eta}{k \mu}}(1+r)^{(n-2 s) q-n-1} d r \\
& \leq C\left(\frac{1}{k \mu}\right)^{(n-2 s) q-n}
\end{aligned}
$$

and

$$
\begin{aligned}
\left\|(1+|y|)^{n+2 s-\frac{2 n}{q}}\right\|_{L^{q}\left\{|y|<\frac{\eta}{k \mu}\right\}}^{q} & \leq C \int_{0}^{\frac{\eta}{k \mu}}(1+r)^{(n+2 s) q-n-1} d r \\
& \leq C\left(\frac{1}{k \mu}\right)^{(n+2 s) q-n}
\end{aligned}
$$

it follows that

$$
\left\|(1+|y|)^{n+2 s-\frac{2 n}{q}} \tilde{E}_{j}(y)\right\|_{L^{q}\left\{|y|<\frac{\eta}{k \mu}\right\}} \leq C k^{-\frac{n}{q}}\left(1+k^{-4 s}\right) .
$$

This gives the proof of (14).

\section{Some linear problems}

Let $L_{0}$ be the linear operator defined by

$$
L_{0}(\phi):=(-\Delta)^{s}(\phi)-p \gamma U^{p-1} \phi \quad \text { in } \mathbb{R}^{n} .
$$


As we know from [Dávila et al. 2013], the set of bounded solutions of the homogeneous equation $L_{0}(\phi)=0$ is spanned by the $n+1$ functions defined by

$$
Z_{i}=\partial_{x_{i}} U, \quad i=1, \ldots, n, \quad \text { and } \quad Z_{n+1}=\frac{1}{2}(n-2 s) U+x \cdot \nabla U .
$$

We now establish a solvability result for the linear problem

$$
L_{0}(\phi)=h \quad \text { in } \mathbb{R}^{n},
$$

under proper orthogonality conditions on $h$ and $\phi$. For this purpose, we introduce the norm

$$
\|\phi\|_{*}:=\left\|\left(1+|y|^{n-2 s}\right) \phi\right\|_{\infty} .
$$

Lemma 3.1. Assume $q \in\left(\frac{n}{2 s}, \frac{n}{s}\right)$. Let $h$ be such that $\|h\|_{* *}<\infty$ and

$$
\int_{\mathbb{R}^{n}} U^{p-1} Z_{l} h d x=0 \quad \text { for all } l=1,2, \ldots, n+1 .
$$

Then the equation

$$
(-\Delta)^{s} \phi-p U^{p-1} \phi=h \quad \text { in } \mathbb{R}^{n}
$$

has a unique solution $\phi$ with $\|\phi\|_{*}<+\infty$ such that

$$
\int_{\mathbb{R}^{n}} U^{p-1} Z_{l} \phi d x=0 \quad \text { for all } l=1,2, \ldots, n+1 .
$$

Furthermore, there exists a constant $C>0$, depending only on $q, s$, and $n$, such

$$
\|\phi\|_{*} \leq C\|h\|_{* *} .
$$

Proof. Let $H^{s}$ be the completion of $C_{0}^{\infty}\left(\mathbb{R}^{n}\right)$ equipped with the norm

$$
\|\phi\|_{H^{s}}=\sqrt{\int_{\mathbb{R}^{n}}|\phi|^{2}+\int_{\mathbb{R}^{n}} \int_{\mathbb{R}^{n}} \frac{|\phi(x)-\phi(y)|^{2}}{|x-y|^{n+2 s}} d x d y,}
$$

and let $\left(H^{s},\langle\cdot, \cdot\rangle_{H^{s}}\right)$ be a Hilbert space with the product

$$
\langle f, g\rangle_{H^{s}}=\int_{\mathbb{R}^{2 n}} \frac{(f(x)-f(y))(g(x)-g(y))}{|x-y|^{n+2 s}} d x d y .
$$

Let us consider the subspace

$$
H=\left\{\phi \in H^{s}\left(\mathbb{R}^{n}\right) \text { such that } \int U^{p-1} Z_{l} \phi d x=0, \quad l=1,2, \ldots, n+1\right\} .
$$

We consider the problem of finding $\phi \in H$ such that

$$
\int_{\mathbb{R}^{n}}(-\Delta)^{\frac{s}{2}} \phi(-\Delta)^{\frac{s}{2}} \tau d x-p \gamma \int_{\mathbb{R}^{n}} U^{p-1} \phi \tau+\int_{\mathbb{R}^{n}} h \tau=0 \quad \text { for all } \tau \in H ;
$$


this variational formulation makes sense if we consider for instance $h \in L^{\frac{2 n}{n+2 s}}$, since $H^{s}\left(\mathbb{R}^{n}\right) \hookrightarrow L^{\frac{2 n}{n-2 s}}\left(\mathbb{R}^{n}\right)$ continuously; see, for instance, [Di Nezza et al. 2012].

Let $f \in L^{\frac{2 n}{n+2 s}}\left(\mathbb{R}^{n}\right)$. By Riesz's theorem there exist a unique $\phi \in H$ such that

$$
\int_{\mathbb{R}^{n}}(-\Delta)^{\frac{s}{2}} \phi(-\Delta)^{\frac{s}{2}} \tau d x+\int_{\mathbb{R}^{n}} f \tau d x=0 \quad \text { for all } \tau \in H .
$$

Thus $A(f)=\phi$ defines a linear operator between $L^{\frac{2 n}{n+2 s}}\left(\mathbb{R}^{n}\right)$ and $H$. By the local compactness of Sobolev embedding [Di Nezza et al. 2012] and the decay at infinity of $U^{p-1}$, we have that the map $H \rightarrow L^{\frac{2 n}{n+2 s}}, \phi \mapsto U^{p-1} \phi$ is compact. Hence, Fredholm's alternative applies to the problem

$$
\phi-A\left(p \gamma U^{p-1} \phi\right)=A(h) .
$$

For $h=0$, we have $L_{0}(\phi)=0$ and $\phi \in H$. Thus $(-\Delta)^{s} \phi=p U^{p-1} \phi$ in $\mathbb{R}^{n}$; hence,

$$
\phi(x)=\sigma_{n, s} p \gamma \int_{\mathbb{R}^{n}} \frac{U^{p-1}(y) \phi(y)}{|x-y|^{n-2 s}},
$$

for some explicit positive constant $\sigma_{n, s}$. We claim that $\phi$ is bounded. Indeed, let $\delta>0$ be a fixed positive small number and write

$$
\int_{\mathbb{R}^{n}} \frac{U^{p-1} \phi(y)}{|x-y|^{n-2 s}}=\int_{|x-y|<\delta} \frac{U^{p-1} \phi(y)}{|x-y|^{n-2 s}}+\int_{|x-y|>\delta} \frac{U^{p-1} \phi(y)}{|x-y|^{n-2 s}}:=I_{1}+I_{2} .
$$

We have

$$
I_{1} \leq C\|\phi\|_{\infty} \int_{|x-y|<\delta} \frac{1}{|x-y|^{n-2 s}} d y \leq C \delta^{2 s}\|\phi\|_{\infty}
$$

and, using the Holder inequality repeatedly,

$$
\begin{aligned}
I_{2} & \leq\left(\int_{|x-y|>\delta}\left(\frac{1}{|x-y|^{n-2 s}}\right)^{\frac{2 n}{n-2 s}}\right)^{\frac{n-2 s}{2 n}}\left(\int_{|x-y|>\delta}\left(U^{p-1} \phi\right)^{\frac{2 n}{n+2 s}}\right)^{\frac{n+2 s}{2 n}} \\
& \leq C\left(\int_{|x-y|>\delta} \phi^{\frac{2 n}{n-2 s}}\right)^{\frac{n-2 s}{2 n}}\left(\int_{|x-y|>\delta} U^{(p-1) \frac{2 n}{4 s}}\right)^{\frac{4 s}{2 n}} \leq C\|\phi\|_{L^{2 n /(n-2 s)}}
\end{aligned}
$$

Choosing $\delta$ properly small, we obtain that $\phi$ is bounded. We can now apply the result in [Dávila et al. 2013] and conclude that $\phi$ is a linear combination of the functions $Z_{l}, l=1, \ldots, n+1$. Since $\phi \in H$ we have that $\phi \equiv 0$. Fredholm's alternative implies that, for any $h$ satisfying the orthogonality condition, a function $\phi \in H$ solution to (29) exists.

Assume now that $\phi$ solves (27), we shall now show the a priori bound (28). We first show that $\phi$ is bounded. First we have

$$
\|\phi\|_{L^{2 n /(n-2 s)}\left(\mathbb{R}^{n}\right)} \leq\|\phi\|_{H^{s}\left(\mathbb{R}^{n}\right)} \leq\|h\|_{L^{2 n /(n-2 s)}\left(\mathbb{R}^{n}\right)} \leq\left\|(1+|y|)^{n+2 s-2 n / q} h\right\|_{L^{q}\left(\mathbb{R}^{n}\right)} .
$$


Observe now that

$$
\phi(x)=\sigma_{n, s} p \int_{\mathbb{R}^{n}} \frac{U^{p-1} \phi(y)}{|x-y|^{n-2 s}}+\sigma_{n, s} \int_{\mathbb{R}^{n}} \frac{h(y)}{|x-y|^{n-2 s}} .
$$

Fixing a small $\delta>0$, we get

$\int_{\mathbb{R}^{n}} \frac{h(y)}{|x-y|^{n-2 s}} d y=\int_{|x-y|<\delta} \frac{h(y)}{|x-y|^{n-2 s}} d y+\int_{|x-y|<\delta} \frac{h(y)}{|x-y|^{n-2 s}} d y=J_{1}+J_{2}$

with

$$
J_{1} \leq \int_{|x-y|<\delta}\left(\frac{1}{|x-y|^{(n-2 s) q^{\prime}}}\right)^{q^{\prime}}\|h\|_{L^{q}\left(\mathbb{R}^{n}\right)} \leq C\|h\|_{L^{q}\left(\mathbb{R}^{n}\right)}
$$

since $q>\frac{n}{2 s}$, and

$$
J_{2} \leq\left(\int_{|x-y|>\delta} \frac{1}{|x-y|^{2 n}}\right)^{\frac{n-2 s}{2 n}}\|h\|_{L^{2 n /(n+2 s)}} \leq C\|h\|_{L^{2 n /(n+2 s)}} .
$$

Thus, thanks also to (30) and (31), for all $x \in \mathbb{R}^{n}$,

$$
|\phi(x)| \leq C \delta^{2 s}\|\phi\|_{\infty}+C\left(\|\phi\|_{L^{2 n /(n-2 s)}\left(\mathbb{R}^{n}\right)}+\|h\|_{L^{q}\left(\mathbb{R}^{n}\right)}+\|h\|_{\left.L^{2 n /(n+2 s)}\right)} .\right.
$$

Choosing $\delta$ small, we conclude that $\phi$ is bounded since

$$
\|\phi\|_{\infty} \leq C\left(\|\phi\|_{L^{2 n /(n-2 s)}\left(\mathbb{R}^{n}\right)}+\|h\|_{L^{q}\left(\mathbb{R}^{n}\right)}+\|h\|_{L^{2 n /(n+2 s)}}\right) .
$$

Next we show the decay rate at infinity of $\phi$. Consider

$$
\tilde{\phi}(y)=|y|^{2 s-n} \phi\left(|y|^{-2} y\right) \text { and } \tilde{h}(y)=|y|^{-n-2 s} h\left(|y|^{-2} y\right) .
$$

A direct computation shows that

$$
(-\Delta)^{s} \tilde{\phi}-p \gamma U^{p-1}(y) \tilde{\phi}=\tilde{h} \quad \text { on } \mathbb{R}^{n} \backslash\{0\},
$$

and

$$
\begin{gathered}
\|\tilde{\phi}\|_{H^{s}\left(\mathbb{R}^{n}\right)}+\|\tilde{\phi}\|_{L^{2 n /(n-2 s)}\left(\mathbb{R}^{n}\right)}=\|\phi\|_{H^{s}\left(\mathbb{R}^{n}\right)}+\|\phi\|_{L^{2 n /(n-2 s)}\left(\mathbb{R}^{n}\right)}, \\
\|\tilde{h}\|_{L^{q}\left(\mathbb{R}^{n}\right)}=\left\|(1+|y|)^{n+2 s-2 n / q} h\right\|_{L^{q}\left(\mathbb{R}^{n}\right)}=\|h\|_{* *} .
\end{gathered}
$$

Applying the estimate (32) to $\tilde{\phi}$, we get

$$
\begin{aligned}
\|\tilde{\phi}\|_{L^{\infty}(B(0,1))} \leq\|\tilde{\phi}\|_{L^{\infty}\left(\mathbb{R}^{n}\right)} & \leq C\left(\|\tilde{\phi}\|_{L^{2 n /(n-2 s)}\left(\mathbb{R}^{n}\right)}+\|\tilde{h}\|_{L^{q}\left(\mathbb{R}^{n}\right)}+\|\tilde{h}\|_{L^{2 n /(n+2 s)}}\right) \\
& \leq C\left(\|\phi\|_{L^{2 n /(n-2 s)}\left(\mathbb{R}^{n}\right)}+\|\tilde{h}\|_{L^{q}\left(\mathbb{R}^{n}\right)}+\|h\|_{L^{2 n /(n+2 s)}}\right) \\
& \leq C\left(\|h\|_{* *}+\|\tilde{h}\|_{L^{q}\left(\mathbb{R}^{n}\right)}\right)=C\|h\|_{* *} .
\end{aligned}
$$

Since $\left\||y|^{n-2 s} \phi\right\|_{L^{\infty}\{|y|>1\}}=\|\tilde{\phi}\|_{L^{\infty}(B(0,1))}$, we conclude that $\|\phi\|_{*} \leq C\|h\|_{* *}$. 
Under further symmetry conditions on $h$ and $\phi$, (27) can be solved without the orthogonality conditions. For a general function $\psi$ defined in $\mathbb{R}^{n}$, consider the symmetries

$$
\psi\left(\bar{y}, y^{\prime}\right)=\psi\left(e^{\frac{2 \pi j}{k} l} \bar{y}, y^{\prime}\right), \quad j=1,2, \ldots, k-1,
$$

and

$$
\psi\left(\bar{y}, \ldots, y_{j}, \ldots, y_{n}\right)=\psi\left(\bar{y}, \ldots,-y_{j}, \ldots, y_{n}\right), \quad j=3, \ldots, n,
$$

together with invariance under the Kelvin transform

$$
\psi(y)=|y|^{2 s-n} \psi\left(|y|^{-2} y\right) .
$$

Lemma 3.2. Assume that $h$ satisfies (33), (34), and $\|h\|_{* *}<\infty$. Furthermore, we assume that

$$
h(y)=|y|^{-n-2 s} h\left(|y|^{-2} y\right) .
$$

Then (27) has a unique bounded solution $\phi=T(h)$ that satisfies symmetries (33), (34), and (35). Moreover, there exists $C$ depending only on $q$, $s$, and $n$ such that

$$
\|\phi\|_{*} \leq\|h\|_{* *} \cdot
$$

The proof of this result is very close to the proof of [del Pino et al. 2011, (4.19)]. We refer the interested reader to that reference.

For a later purpose, we need to establish a result like the one in Lemma 3.1 for a linear operator more general then $L_{0}$.

Lemma 3.3. Let $2 s<v<n$. There exist numbers $\delta, C$, depending on $v, n$ such that the following holds: If $g, a$, and $\phi$ are functions such that $\left\|\left(1+|y|^{v}\right) g\right\|_{\infty}<+\infty$, $\left\|\left(1+|y|^{\nu-2 s}\right) \phi\right\|_{\infty}<+\infty$, and $\left\|\left(1+|y|^{2 s}\right) a\right\|_{\infty}<\delta$, and

$$
L_{0}(\phi)+a(y) \phi=g(y)+\sum_{l=1}^{n+1} c_{l} U^{p-1} Z_{l} \quad \text { in } \mathbb{R}^{n},
$$

where

$$
\int_{\mathbb{R}^{n}} U^{p-1} Z_{l} \phi=0 \quad \text { for all } l=1, \ldots, n+1
$$

and

$$
c_{l} \int_{\mathbb{R}^{n}} U^{p-1} Z_{l}^{2}=\int_{R^{n}}(a(y) \phi-g(y)) Z_{l} \phi \quad \text { for all } l=1, \ldots, n+1,
$$

then

$$
\left\|\left(1+|y|^{\nu-2 s}\right) \phi\right\|_{\infty} \leq C\left\|\left(1+|y|^{\nu}\right) g\right\|_{\infty} .
$$


Proof. By contradiction, let us assume the existence of functions $\phi_{n}, a_{n}, g_{n}$ and constants $c_{l}^{n}$ such that (36)-(38) hold, and

$$
\left\|\left(1+|y|^{\nu}\right) g_{n}\right\|_{\infty} \rightarrow 0, \quad\left\|\left(1+|y|^{\nu-2 s}\right) \phi_{n}\right\|_{\infty}=1, \quad\left\|\left(1+|y|^{2 s}\right) a_{n}\right\|_{\infty} \rightarrow 0 .
$$

Clearly, $\left\|\left(1+|y|^{\nu}\right) a_{n} g_{n}\right\|_{\infty} \rightarrow 0$ and $c_{l}^{n} \rightarrow 0$, so without loss of generality we may assume that $a_{n} \equiv 0$ and $c_{l}^{n}=0$. We claim first that

$$
\left\|\phi_{n}\right\|_{\infty} \rightarrow 0 \text {. }
$$

Assume the opposite: there are numbers $\gamma, R>0$ and points $x_{n}$ such that

$$
\left|\phi_{n}\left(x_{n}\right)\right| \geq \gamma, \quad\left|x_{n}\right| \leq R .
$$

Passing to a subsequence, and arguing like in the proof of Lemma 3.1, we find that $\phi_{n}$ converges in the energy space and locally uniformly over compact sets to a bounded function $\phi_{0} \neq 0$ with

$$
L_{0}\left(\phi_{0}\right)=0, \quad \text { and } \quad \int_{\mathbb{R}^{n}} U^{p-1} \phi Z_{l}=0 \text { for all } l,
$$

which gives $\phi_{0}=0$. This is a contradiction due to the result in [Dávila et al. 2013]. Thus we have that $\left\|\phi_{n}\right\|_{\infty} \rightarrow 0$.

Next we shall show that $\left\|\left(1+|y|^{\nu-2 s}\right) \phi_{n}\right\|_{\infty} \rightarrow 0$, thus getting to a contradiction with (40), and the proof of the Lemma. Using the equation, we have that

$$
\phi_{n}(x)=\sigma_{n, s} p \gamma \int_{\mathbb{R}^{n}} \frac{U^{p-1}(y) \phi_{n}(y)}{|x-y|^{n-2 s}} d y+\sigma_{n, s} \int_{\mathbb{R}^{n}} \frac{g_{n}(y)}{|x-y|^{n-2 s}} d y
$$

for some explicit positive constant $\sigma_{n, s}$. Since $2 s<v<n$, and taking into account that $\left\|\left(1+|y|^{v}\right) g_{n}\right\|_{\infty} \rightarrow 0$, as well as the behavior of $U^{p-1}$ at infinity, there exists a positive constant $C$, independent of $n$, such that

$$
\left|\phi_{n}(x)\right| \leq C\left(\frac{\left\|\phi_{n}\right\|_{\infty}}{\left(1+|x|^{2 s}\right)}+\frac{o(1)}{\left(1+|x|^{\nu-2 s}\right)}\right)
$$

for some $o(1) \rightarrow 0$, as $n \rightarrow \infty$. Replacing the above estimate in (41) and repeating the same procedure a finite number of times, we get that

$$
\left|\phi_{n}(x)\right| \leq C \frac{\left\|\phi_{n}\right\|_{\infty}+o(1)}{\left(1+|x|^{\nu-2 s}\right)} .
$$

\section{Proof of Propositions 2.1 and 2.2}

Proof of Proposition 2.1. Let us fix functions $\tilde{\phi}_{j}$ and we assume that they satisfy the symmetry assumptions (6), (7) and the invariance under Kelvin transform

$$
\tilde{\phi}_{1}=|y|^{2 s-n} \tilde{\phi}_{1}\left(|y|^{-2} y\right) \text {. }
$$


Finally, we assume

$$
\left\|\phi_{1}\right\|_{*}<\rho, \quad \text { where } \phi_{1}=\mu^{\frac{n-2 s}{2}} \tilde{\phi}_{1}\left(\xi_{1}+\mu y\right) .
$$

for a small, fixed $\rho>0$.

We next solve (17). To do so, we write it in the form

$$
(-\Delta)^{s}(\psi)-p \gamma U^{p-1}(y) \psi-\underbrace{\gamma V(y) \psi-p \gamma\left|U_{*}\right|^{p-1} \sum_{j=1}^{k}\left(1-\zeta_{j}\right) \tilde{\phi}_{j}-M(\psi)}_{:=h}=0,
$$

where

$$
V(y):=\underbrace{p\left(\left|U_{*}\right|^{p-1}-U^{p-1}\right)\left(1-\sum_{j=1}^{k} \zeta_{j}\right)}_{:=V_{1}}+\underbrace{p U^{p-1} \sum_{j=1}^{k} \zeta_{j}}_{:=V_{2}}:=V_{1}+V_{2}
$$

and

$$
M(\psi):=\left(1-\sum_{j=1}^{k} \zeta_{j}\right)\left(E+\gamma N\left(\sum_{j=1}^{k} \tilde{\phi}_{j}+\psi\right)\right)
$$

A basic observation is that the function $h$ as defined above satisfies the conditions (33), (34), and $\|h\|_{* *}<\infty$. Furthermore, we have that

$$
h(y)=|y|^{-n-2 s} h\left(|y|^{-2} y\right) .
$$

Hence, we can define the linear operator $T$ in the Lemma 3.2 and we can write our problem (17) in fixed point as

$$
\psi=-T\left(V \psi+p \gamma\left|U_{*}\right|^{p-1} \sum_{j}(1-\zeta) \tilde{\phi}_{j}+M(\psi)\right)=: \mathcal{M}(\psi)
$$

We notice that $\mathcal{M}$ is well defined in space $X$ of continuous functions $\psi$ with $\|\psi\|_{*} \leq \infty$, and satisfying

$$
\begin{array}{rlrl}
\psi\left(\bar{y}, y^{\prime}\right) & =\psi\left(e^{\frac{2 \pi j}{k} l} \bar{y}, y^{\prime}\right), & j & =1,2, \ldots, k-1, \\
\psi\left(\bar{y}, \ldots, y_{j}, \ldots, y_{n}\right) & =\psi\left(\bar{y}, \ldots,-y_{j}, \ldots, y_{n}\right), & j=3, \ldots, n, \\
\psi & =|y|^{2 s-n} \psi\left(|y|^{-2} y\right) . & &
\end{array}
$$

We claim that

$$
\|V \psi(y)\|_{* *} \leq C k^{1-\frac{n}{q}}\|\psi\|_{*}
$$

and

$$
\left\|p \gamma\left|U_{*}\right|^{p-1} \sum_{j=1}^{k}\left(1-\zeta_{j}\right) \tilde{\phi}_{j}\right\|_{* *} \leq C k^{1-\frac{n}{q}}\|\psi\|_{*} .
$$


We claim that if

$$
\|\psi\|_{*}+\left\|\phi_{1}\right\|_{*} \leq 2 \rho,
$$

then

$$
\|M(\psi)\|_{* *} \leq C\left(k^{1-\frac{n}{q}}+k^{1-\frac{n}{q}}\left\|\phi_{1}\right\|_{*}^{2}+\|\psi\|_{*}^{2}\right) .
$$

Furthermore, for $\psi_{1}, \psi_{2}$ in $X$,

$$
\left\|M\left(\psi_{1}\right)-M\left(\psi_{2}\right)\right\|_{* *} \leq C \rho\left\|\psi_{1}-\psi_{2}\right\|_{*}
$$

We can thus conclude that, for $\rho$ small enough, the operator $\mathcal{M}$ defines a contraction map in the set of functions $\psi \in X$ with

$$
\|\psi\|_{*} \leq C\left(\left\|\phi_{1}\right\|_{*}^{2}+k^{1-\frac{n}{q}}\right) .
$$

From the estimate (47), we get the Lipschitz dependence

$$
\left\|\Psi\left(\phi_{1}^{1}\right)-\Psi\left(\phi_{1}^{2}\right)\right\|_{*} \leq C\left\|\phi_{1}^{1}-\phi_{1}^{2}\right\|_{*} .
$$

We shall next show the validity of (44), (45), and (46).

Proof of (44). Consider

$$
f(t)=\left|U-t \sum_{j=1}^{k} U_{j}\right|^{p-1} .
$$

By the mean value theorem,

$$
\left|V_{1}\right| \leq p(p-1)\left|U-s \sum_{j=1}^{k} U_{j}\right|^{p-2}\left(\sum_{j=1}^{k} U_{j}\right) \leq C U^{p-2} \sum_{j=1}^{k} \frac{\mu^{\frac{n-2 s}{2}}}{\left|y-\xi_{j}\right|^{n-2 s}} .
$$

Thus, if for all $j,\left|y-\xi_{j}\right|>\frac{\eta}{k}$, then

$$
\left|V_{1} \psi(y)\right| \leq C\|\psi\|_{*} U^{p-1}(y) \sum_{j=1}^{k} \frac{\mu^{\frac{n-2 s}{2}}}{\left|y-\xi_{j}\right|^{n-2 s}} .
$$

Since $\zeta_{j} \equiv 1$ on $\left|y-\xi_{j}\right|<\frac{\eta}{k}$,

$$
\begin{aligned}
\left\|(1+|y|)^{n+2 s-\frac{2 n}{q}} V_{1} \psi\right\|_{L^{q}\left(\mathbb{R}^{n}\right)} & =\left\|(1+|y|)^{n+2 s-\frac{2 n}{q}} V_{1} \psi\right\|_{L^{q}\left(\mathbb{R}^{n} \backslash \bigcup_{j} B\left(\xi_{j}, \frac{\eta}{k}\right)\right)} \\
& \leq C k\left(\int_{B\left(\xi_{1}, \frac{\eta}{k}\right) c \cap B(0,2)} \frac{\mu^{\frac{(n-2 s) q}{2}}}{\left|y-\xi_{j}\right|^{(n-2 s) q}} d y\right)^{\frac{1}{q}}\|\psi\|_{*} \\
& \leq C k \mu^{\frac{n-2 s}{2}} k^{(n-2 s)-\frac{n}{q}}\|\psi\|_{*},
\end{aligned}
$$


for some positive constant $C$. Thus $\left\|V_{1} \psi(y)\right\|_{* *} \leq C k^{1-\frac{n}{q}}\|\psi\|_{*}$. On the other hand,

$$
\begin{aligned}
\left\|V_{2} \psi\right\|_{* *} & =\left\|(1+|y|)^{n+2 s-\frac{2 n}{q}} p U^{p-1} \sum_{j=1}^{k} \zeta_{j} \psi\right\|_{L^{q}\left(\mathbb{R}^{n}\right)} \\
& \leq C\left(\int_{B(0,1)}\left((1+|y|)^{n+2 s-\frac{2 n}{q}} U^{p-1} \sum_{j=1}^{k} \zeta_{j} \psi\right)^{q} d y\right)^{\frac{1}{q}}
\end{aligned}
$$

with

$$
\begin{aligned}
&\left(\int _ { B ( 0 , 1 ) } \left((1+|y|)^{n+2 s-\frac{2 n}{q}}\right.\right.\left.\left.U^{p-1} \sum_{j=1}^{k} \zeta_{j} \psi\right)^{q} d y\right)^{\frac{1}{q}} \\
& \leq C \sum_{j}\left(\int_{B\left(\xi_{j}, \frac{2 \eta}{k}\right)} \frac{U^{(p-1) q}(1+|y|)^{(n+2 s) q-2 n}}{(1+|y|)^{(n-2 s) q}} d y\right)^{\frac{1}{q}}\|\psi\|_{*} \\
& \leq C k^{1-n}\|\psi\|_{*}
\end{aligned}
$$

Proof of (45). Estimate (45) can be obtained arguing as in the proof of estimate (44), after noticing that

$$
\left|\tilde{\phi}_{j}(y)\right| \leq C U(y)\left\|\phi_{1}\right\|_{*} \frac{\mu^{\frac{n-2 s}{2}}}{|y-\xi|^{n-2 s}} .
$$

Proof of (46). For the moment we shall assume that

$$
\|\psi\|_{*}+\left\|\phi_{1}\right\|_{*} \leq 2 \rho
$$

for a $\rho$ sufficiently small. Let us assume that $\left|y-\xi_{j}\right|>\frac{\eta}{k}$ for all $j$. First we recall that

$$
\left\|(1+|y|)^{n+2 s-\frac{2 n}{q}}\left(1-\sum_{j=1}^{k} \zeta_{j}\right) E\right\|_{L^{q}\left(\mathbb{R}^{n}\right)}=\left\|(1+|y|)^{n+2 s-\frac{2 n}{q}} E\right\|_{L^{q}(\mathrm{Ext})} \leq C k^{1-\frac{n}{q}}
$$

Then we find in this region

$$
\left|N\left(\sum_{j=1}^{k} \tilde{\phi}_{j}+\psi\right)\right| \leq C U^{p-2}\left(\left|\sum_{j=1}^{k} \tilde{\phi}_{j}\right|^{2}+|\psi|^{2}\right) .
$$

But

$$
U^{p-2}\left|\sum_{j=1}^{k} \tilde{\phi}_{j}\right|^{2} \leq C\left\|\phi_{1}\right\|_{*}^{2} U^{p} \sum_{j=1}^{k} \frac{\mu^{n-2 s}}{\left|y-\xi_{j}\right|^{2(n-2 s)}}, \quad U^{p-2}|\psi|^{2} \leq U^{p}\|\psi\|_{*}^{2}
$$


Thus, we have

$$
\begin{aligned}
\|(1+|y|)^{n+2 s-\frac{2 n}{q}} & \left(1-\sum_{j=1}^{k} \zeta_{j}\right)\left(\gamma N\left(\sum_{j=1}^{k} \tilde{\phi}_{j}+\psi\right)\right) \|_{L^{q}\left(\mathbb{R}^{n}\right)} \\
& =\left\|(1+|y|)^{n+2 s-\frac{2 n}{q}}(\gamma N(\phi))\right\|_{L^{q}(\mathrm{Ext})} \\
& \leq C\left\|\phi_{1}\right\|_{*}^{2}\left\|(1+|y|)^{n+2 s-\frac{2 n}{q}} U^{p}\left(\sum_{j=1}^{k} \frac{\mu^{n-2 s}}{\left|y-\xi_{j}\right|^{2(n-2 s)}}+\psi\right)\right\|_{L^{q}(\mathrm{Ext})} \\
& \leq \frac{C \mu^{n-2 s}}{k^{-2(n-2 s)+\frac{n}{q}-1}}\left\|\phi_{1}\right\|_{*}^{2}+C\|\psi\|_{*}^{2}
\end{aligned}
$$

Using the above inequalities, we get

$$
\|M(\psi)\|_{* *} \leq C k^{1-\frac{n}{q}}+k^{1-\frac{n}{q}}\left\|\phi_{1}\right\|_{*}^{2}+C\|\psi\|_{*}^{2},
$$

This concludes the proof of Proposition 2.1.

Proof of Proposition 2.2. In order to prove Proposition 2.2, we need to consider the linear problem

$$
(-\Delta)^{s} \tilde{\phi}_{1}-p \gamma U_{1}^{p-1} \tilde{\phi}-\tilde{h}(y)=c_{n+1} U_{1}^{p-1} \tilde{Z}_{n+1} \quad \text { in } \mathbb{R}^{n}
$$

for a general function $\tilde{h}$, where

$$
\tilde{Z}_{n+1}(y)=\mu^{-\frac{n-2 s}{2}} Z_{n+1}\left(\mu^{-1}\left(y-\xi_{1}\right)\right) \quad \text { and } \quad c_{n+1}=\frac{\int_{\mathbb{R}^{n}} \tilde{h} \tilde{Z}_{n+1}}{\int_{\mathbb{R}^{n}} U_{1}^{p-1} \tilde{Z}_{n+1}^{2}} .
$$

Lemma 4.1. Assume that $\tilde{h}$ is even with respect to each variable $y_{2}, \ldots, y_{n}$ and it satisfies the invariance

$$
\tilde{h}(y)=|y|^{-n-2 s} h\left(|y|^{-2} y\right)
$$

Assume in addition that

$$
h(y)=\mu^{\frac{n+2 s}{2}} \tilde{h}\left(\xi_{1}+\mu y\right)
$$

satisfies $\|h\|_{* *} \leq \infty$. Then (48) has a unique solution $\tilde{\phi}:=\tilde{T}(\tilde{h})$ that is even with respect to each of the variables $y_{2}, \ldots, y_{n}$, invariant under Kelvin's transformations

$$
\tilde{\phi}(y)=|y|^{2 s-n} \tilde{\phi}\left(|y|^{-2} y\right),
$$

where $\phi(y)=\mu^{\frac{n-2 s}{2}} \tilde{\phi}\left(\xi_{1}+\mu y\right)$ and satisfies

$$
\int_{\mathbb{R}^{n}} \phi U^{p-1} Z_{n+1}=0
$$


Moreover, there exists $C$ such that

$$
\|\phi\|_{*} \leq C\|h\|_{* *} .
$$

Proof. We consider $\phi$ and $h$ such that

$$
(-\Delta)^{s} \phi-p \gamma|U|^{p-1} \phi=h(y) \quad \text { in } \mathbb{R}^{n}, \quad \text { and } \quad \int_{\mathbb{R}^{n}} \tilde{h} \tilde{Z}_{n+1}=0 .
$$

The evenness of $h$ in the last $(n-1)$ coordinates guarantees that

$$
\int_{\mathbb{R}^{n}} h Z_{l}=0, \quad l=2, \ldots, n, n+1 .
$$

We have that to prove that $\int_{\mathbb{R}^{n}} h Z_{1}=0$. Let

$$
I(t)=\int_{\mathbb{R}^{n}} w_{\mu}\left(y-t \xi_{1}\right) \tilde{h}(y) d y .
$$

We notice that

$$
\left(\xi_{1}\right)_{1} \int_{\mathbb{R}^{n}} h Z_{1}=\left.\partial_{t} I(t)\right|_{t=0}=-\left(\xi_{1}\right)_{1} \int_{\mathbb{R}^{n}} \partial_{y_{1}} w_{\mu}\left(y-\xi_{1}\right) h(y) d y ;
$$

after a change of variable,

$$
I(t)=\int_{\mathbb{R}^{n}} w_{\mu}\left(|y|^{-2} y-t \xi_{1}\right) \tilde{h}\left(|y|^{-2} y\right)|y|^{-2 n}=\int_{\mathbb{R}^{n}} w_{\mu(t)}\left(y-a(t) \xi_{1}\right) \tilde{h}(y) d y
$$

where

$$
\mu(t)=\frac{\mu t}{\mu^{2}+\left|\xi_{1}\right| 2 t^{2}} \quad \text { and } \quad s(t)=\frac{t}{\mu^{2}+\left|\xi_{1}\right|^{2} t^{2}}
$$

Hence,

$$
\begin{aligned}
\left.\partial_{t} I(t)\right|_{t=1}=\left.\mu^{\prime}(1) \int_{\mathbb{R}^{n}} \partial_{\mu} w_{\mu}\left(y-\xi_{1}\right)\right|_{\mu=1} \tilde{h}(y) d y & \\
& -s^{\prime}(1) \xi_{1} \int_{\mathbb{R}^{n}} \partial_{y_{1}} w_{\mu}(t)\left(y-\xi_{1}\right) h(y) d y=0 .
\end{aligned}
$$

We can check that

$$
\left.\int_{\mathbb{R}^{n}} \partial_{\mu} w_{\mu}\left(y-\xi_{1}\right)\right|_{\mu=1} \tilde{h}(y) d y=\int_{\mathbb{R}^{n}} Z_{n+1}(y) h(y) d y=0
$$

and $s^{\prime}(1)=1-2\left|\xi_{1}\right|^{2}$. Hence, using (49) and (50), we obtain $\int_{\mathbb{R}^{n}} h Z_{1}=0$. It follows from Lemma 3.1 that there exists a unique solution $\phi_{1}$ for (48) with

$$
\int_{\mathbb{R}^{n}} h Z_{l}=0, \quad l=1, \ldots, n+1 \quad \text { and } \quad\|\phi\|_{*} \leq C\|h\|_{* *} \cdot
$$

Arguing by uniqueness, as in proof of Lemma 3.2, we find that $\tilde{\phi}$ satisfies the corresponding symmetries. 
We use the above lemma to solve (23) and (24). We consider the operator $\tilde{T}$ defined in the lemma. We are going to prove the existence of a solution to (23) by a fixed point argument

$$
\tilde{\phi}_{1}=\tilde{T}\left(\zeta_{1}+\gamma \mathcal{N}\left(\phi_{1}\right)\right)=: \mathcal{M}\left(\phi_{1}\right) .
$$

For any $f$ we set $\bar{f}(y)=\mu^{\frac{n+2 s}{2}} f(\xi+\mu y)$. Let

$$
f_{1}(y)=p \zeta_{1}\left(\left|U_{*}\right|^{p-1}-\left|U_{1}\right|^{p-1}\right) \tilde{\phi}_{1} .
$$

For $|y|<\frac{\eta}{k \mu}$,

and so

$$
\left|\bar{f}_{1}(y)\right| \leq C\left(\mu^{n-2 s} k^{n-2 s} \sum_{j=1}^{k-1} \frac{1}{j^{n-2 s}}+\mu^{\frac{n-2 s}{2}}\right) U^{p-1}\left\|\phi_{1}\right\|_{*}
$$

$$
\left\|\bar{f}_{1}(y)\right\|_{* *} \leq C\left(\mu^{n-2 s} k^{n-2 s}+\mu^{\frac{n-2 s}{2}}\right)(\mu k)^{-n+2 s+\frac{n}{q}}\left\|\phi_{1}\right\|_{*}=C \mu^{\frac{2 n}{q}}\left\|\phi_{1}\right\|_{*} .
$$

Analogously for $f_{2}=\left(\zeta_{1}-1\right) U_{1}^{p-1} \tilde{\phi}_{1}$ in the region $|y|<\frac{\eta}{\mu k}$,

$$
\left|\bar{f}_{2}(y)\right| \leq U^{p}\left\|\phi_{1}\right\|_{*} ;
$$

hence $\left\|\bar{f}_{2}\right\|_{* *} \leq C k^{-\frac{n}{q}}\left\|\phi_{1}\right\|_{*}$. Now we consider $f_{3}=\zeta_{1} p\left|U_{*}\right|^{p-1} \Psi\left(\phi_{1}\right)$ on $|y|<\frac{n}{\mu k}$,

$$
\left|\bar{f}_{3}\right| \leq C U^{p-1} \mu^{\frac{n-2 s}{2}}\left\|\Psi\left(\phi_{1}\right)\right\|_{\infty} \leq C U^{p-1} \mu^{\frac{n-2 s}{2}}\left(\left\|\phi_{1}\right\|_{*}+k^{1-\frac{n}{q}}\right) ;
$$

thus,

$$
\left\|\bar{f}_{3}(y)\right\|_{* *} \leq C \mu^{\frac{n}{2 q}}\left(\left\|\phi_{1}\right\|_{*}+k^{1-\frac{n}{q}}\right) .
$$

Now, for

$$
f_{4}=\zeta_{1} N\left(\tilde{\phi}_{1}+\sum_{i=2} \tilde{\phi}_{i}\right) \Psi\left(\phi_{1}\right)
$$

we notice that

$$
\bar{N}(\phi)=\left(V_{*}+\hat{\phi}\right)^{p}-V_{*}^{p}-p V_{*}^{p-1} \hat{\phi}
$$

where $\hat{\phi}(y):=\mu^{\frac{n-2 s}{2}} \phi\left(\xi_{1}+\mu y\right)$ and

$$
V_{*}(y)=U(y)+\sum_{i=2}^{k} U\left(y+\mu^{-1}\left(\xi_{1}-\xi_{j}\right)\right)-\mu^{\frac{n-2 s}{2}} U\left(\xi_{1}+\mu y\right)
$$

with

$$
\phi=\tilde{\phi}_{1}+\sum_{i=2}^{k} \tilde{\phi}_{i}+\Psi\left(\phi_{1}\right)
$$

Therefore

$$
\left|\bar{f}_{4}\right| \leq C\left(U^{p-1} \mu^{\frac{n-2 s}{2}}\left\|\phi_{1}\right\|_{*}+U^{p-1} \mu^{\frac{n-2 s}{2}}\left(\left\|\phi_{1}\right\|_{*}+k^{1-\frac{n}{q}}\right)\right),
$$


and hence,

$$
\left\|\bar{f}_{4}\right\|_{* *} \leq C\left(\mu^{\frac{n}{2 q}}\left\|\phi_{1}\right\|_{*}+\mu^{\frac{n}{2 q}}\left(\left\|\phi_{1}\right\|_{*}+k^{1-\frac{n}{q}}\right)^{2}\right) .
$$

Concerning $f_{5}=\zeta_{1} E$, we recall that

$$
\left\|\bar{f}_{5}\right\|_{* *} \leq C \mu^{\frac{n}{2 q}} .
$$

The above estimates suggest that it is possible to apply a fixed point argument of contraction type in the set of all continuous functions $\phi_{1}=\Phi(\delta)$ such that $\left\|\phi_{1}\right\|_{*} \leq C \mu^{\frac{n}{2 q}}$. This gives the existence and the estimate for $\phi_{1}$, satisfying

$$
\|\Phi\|_{*} \leq C k^{-\frac{n}{q}}
$$

and

$$
\|\mathcal{N}(\phi)\|_{* *} \leq C k^{-\frac{2 n}{q}} \text {. }
$$

Straightforward computations shows also the continuous dependence of $\phi_{1}=\Phi(\delta)$ and $c_{n+1}$ on the parameter $\delta$. This concludes the proof of Proposition 2.2.

\section{Conclusion}

In this section we show the existence of $\delta>0$ such that $c_{n+1}(\delta)=0$ in (23). Indeed this fact guarantees that the function

$$
U_{*}+\phi,
$$

where $U_{*}=U-\sum U_{j}$ is defined in (9) and $\phi=\sum_{j=1}^{k} \tilde{\phi}_{j}+\psi$ is defined in (15), is a solution for the original problem (1). Let

$$
\tilde{Z}_{n+1}=\mu^{-\frac{n-2 s}{2}} Z_{n+1}\left(\mu^{-1}\left(y-\xi_{1}\right)\right) .
$$

We recall that

$$
Z_{n+1}(y)=y \cdot \nabla U+\frac{n-2 s}{2} U .
$$

We need the existence of a $\delta$ such that

$$
c_{n+1}=\int_{\mathbb{R}^{n}}\left(\zeta_{1} E+\gamma \mathcal{N}\left(\phi_{1}\right)\right) \tilde{Z}_{n+1}=0 .
$$

Since we are assuming that $s>\frac{1}{2}$, we claim that

$$
\int_{\mathbb{B}^{n}} \zeta_{1} E \tilde{Z}_{n+1}=A \delta k^{2 s-n}\left(-2^{\frac{n-2 s}{2}}\left(\sum_{j=1}^{\infty} \frac{1}{j^{n-2 s}}\right) \delta+1\right)+k^{1-n} \Theta_{k}(\delta)
$$

and

$$
\int_{\mathbb{R}^{n}} \gamma \mathcal{N}\left(\phi_{1}\right) \tilde{Z}_{n+1}=k^{-(n-2 s)} k^{1-\frac{n}{q}} \Theta_{k}(\delta),
$$


where $\Theta_{k}(\delta)$ denotes a continuous function of $\delta$, which is uniformly bounded, as $k \rightarrow \infty$. Since $n-2 s>1$ for any $s \in\left(\frac{1}{2}, 1\right)$, from (53) and (54) we obtain the existence of a unique $\delta$ solution to (52) with

$$
\delta=\left(2^{\frac{n-2 s}{2}}\left(\sum_{j=1}^{\infty} \frac{1}{j^{n-2 s}}\right)\right)^{-1}\left(1+O\left(k^{1-2 s}\right)\right) .
$$

What is left of this section is devoted to the proof of (53) and (54).

Proof of (53). We write

$$
\int_{\mathbb{R}^{n}} \zeta_{1} E \tilde{Z}_{n+1}=\int_{\mathbb{R}^{n}} E \tilde{Z}_{n+1}+\int_{\mathbb{R}^{n}}\left(\zeta_{1}-1\right) E \tilde{Z}_{n+1}
$$

Expanding the first term, we get

$$
\int_{\mathbb{R}^{n}} E \tilde{Z}_{n+1}=\int_{B_{1}} E \tilde{Z}_{n+1}+\int_{\mathbb{R}^{n} \backslash \cup B_{j}} E \tilde{Z}_{n+1}+\sum_{j \neq 1} \int_{B_{j}} E \tilde{Z}_{n+1}:=I_{1}+I_{2}+I_{3},
$$

where $B_{j}=B\left(\xi_{j}, \frac{\eta}{k}\right)$. With the scaling $x=\mu y+\xi_{1}$ and writing

$$
\tilde{E}(y)=\mu^{\frac{n+2 s}{2}} E\left(\xi_{1}+\mu y\right),
$$

we get

$$
\int_{B_{1}} E \tilde{Z}_{n+1}=\int_{B\left(0, \frac{\eta}{\mu k}\right)} \tilde{E}_{1}(y) Z_{n+1}(y) d y .
$$

Thus

$$
\begin{aligned}
I_{1}= & \int_{B\left(0, \frac{\eta}{\mu k}\right)} \tilde{E}_{1} Z_{n+1}(y) d y \\
= & \gamma p \sum_{j \neq 1} \int_{B\left(0, \frac{\eta}{\mu k}\right)} U^{p-1} U\left(y-\mu^{-1}\left(\xi_{j}-\xi_{1}\right)\right) Z_{n+1} \\
& +\gamma p \mu^{\frac{n-2 s}{2}} \int_{B\left(0, \frac{\eta}{\mu k}\right)} U^{p-1} U\left(\xi_{1}+\mu y\right) Z_{n+1} d y \\
& +\gamma p \int_{B\left(0, \frac{\eta}{\mu k}\right)}\left((U(y)+s V(y))^{p-1}-U^{p-1}\right) V(y) Z_{n+1} d y \\
& +\gamma \sum_{j \neq 1} \int_{B\left(0, \frac{\eta}{\mu k}\right)} U^{p}\left(y-\mu^{-1}\left(\xi_{j}-\xi_{1}\right)\right) Z_{n+1} \\
& -\mu^{\frac{n+2 s}{2}} \gamma \int_{B\left(0, \frac{\eta}{\mu k}\right)} U^{p}\left(\xi_{j}+\mu y\right) Z_{n+1} d y,
\end{aligned}
$$


where

$$
V(y)=\left(-\sum_{j \neq 1} U\left(y-\mu^{-1}\left(\xi_{j}-\xi_{1}\right)\right)+\mu^{\frac{n-2 s}{2}} U\left(\xi_{1}+\mu y\right)\right) .
$$

For $j \neq 1$, and by Taylor expansion,

$$
U\left(y+\mu^{-1}\left(\xi_{1}-\xi_{j}\right)\right)=\frac{2^{\frac{n-2 s}{2}} \mu^{n-2 s}}{\left|\hat{\xi}_{j}-\hat{\xi}_{1}\right|^{n-2 s}}\left(1+O\left(\mu^{2} k^{2}\right)\right),
$$

where $\hat{\xi}_{1}=(1,0, \ldots, 0)$ and

$$
\hat{\xi}_{j}=e^{\frac{2 \pi(j-1)}{k}} \hat{\xi}_{1}
$$

thus

$$
\begin{aligned}
\int_{B\left(0, \frac{\eta}{\mu k}\right)} U^{p-1} U\left(y-\mu^{-1}\left(\xi_{j}\right.\right. & \left.\left.-\xi_{1}\right)\right) Z_{n+1} \\
= & \frac{2^{\frac{n-2 s}{2}} \mu^{n-2 s}}{\left|\hat{\xi}_{j}-\hat{\xi}_{1}\right|^{n-2 s}} \int_{B\left(0, \frac{\eta}{\mu k}\right)} U^{p-1}\left(1+O\left(\mu^{2} k^{2}\right)\right) Z_{n+1} \\
= & \frac{2^{\frac{n-2 s}{2}} \mu^{n-2 s}}{\left|\hat{\xi}_{j}-\hat{\xi}_{1}\right|^{n-2 s}}\left(\int_{\mathbb{R}^{n}} U^{p-1} Z_{n+1}-\int_{\mathbb{R}^{n} \backslash B\left(0, \frac{\eta}{\mu k}\right)} U^{p-1} Z_{n+1}\right. \\
& \left.+O\left(\mu^{2} k^{2}\right) \int_{B\left(0, \frac{\eta}{\mu k}\right)} U^{p-1} Z_{n+1}\right) \\
= & \frac{2^{\frac{n-2 s}{2}} \mu^{n-2 s}}{\left|\hat{\xi}_{j}-\hat{\xi}_{1}\right|^{n-2 s}}\left(C_{1}+O\left(\mu^{2 s} k^{2 s}\right)+O\left(\mu^{2} k^{2}\right)\right) \\
= & \frac{2^{\frac{n-2 s}{2}} \mu^{n-2 s}}{\left|\hat{\xi}_{j}-\hat{\xi}_{1}\right|^{n-2 s}} C_{1}\left(1+O\left(\mu^{2 s} k^{2 s}\right)\right),
\end{aligned}
$$

where

$$
C_{1}=\int_{\mathbb{R}^{n}} U^{p-1} Z_{n+1}
$$

For the second term,

$$
\mu^{\frac{n-2 s}{2}} \int_{B\left(0, \frac{\eta}{\mu k}\right)} U^{p-1} U\left(\xi_{1}+\mu y\right) Z_{n+1} d y=\mu^{\frac{n-2 s}{2}} C_{1}\left(1+O\left(\mu^{2 s} k^{2 s}\right)\right)
$$


Furthermore,

$$
\begin{aligned}
\mid \int_{B\left(0, \frac{\eta}{\mu k}\right)}\left((U(y)+s V(y))^{p-1}-U^{p-1}\right) & V(y) Z_{n+1} d y \mid \\
\leq & \left|\sum_{i \neq 1} \int_{B\left(0, \frac{\eta}{\mu k}\right)} U^{p}\left(y-\mu^{-1}\left(\xi_{j}-\xi_{1}\right)\right) Z_{n+1}\right| \\
& \leq C \sum_{i \neq 1} \frac{\mu^{n+2 s}}{\left|\hat{\xi}_{1}-\hat{\xi}_{i}\right|^{n+2 s}} \int_{B\left(0, \frac{\eta}{\mu k}\right)} \frac{1}{(1+|y|)^{n-2 s}} \\
& \leq C(\mu k)^{-2 s} \sum_{i \neq 1} \frac{\mu^{n+2 s}}{\left|\hat{\xi}_{1}-\hat{\xi}_{i}\right|^{n+2 s}}
\end{aligned}
$$

and

$$
\begin{aligned}
& \left|\mu^{\frac{n+2 s}{2}} \gamma \int_{B\left(0, \frac{\eta}{\mu k}\right)} U^{p}\left(\xi_{j}+\mu y\right) Z_{n+1} d y\right| \\
& \quad \leq C \mu^{\frac{n+2 s}{2}} \int_{B\left(0, \frac{\eta}{\mu k}\right)} \frac{1}{(1+|y|)^{n-2 s}} d y \leq C \mu^{\frac{n-2 s}{2}} k^{-2 s} .
\end{aligned}
$$

Therefore, we conclude that

$$
I_{1}=A \delta k^{-(n-2 s)}\left(-2^{\frac{n-2 s}{2}}\left(\sum_{j=1}^{\infty} \frac{1}{j^{n-2 s}}\right) \delta+1\right)+k^{-n} \Theta_{k}(\delta),
$$

where $\Theta_{k}(\delta)$ is a smooth function of $\delta$, which is uniformly bounded as $k \rightarrow \infty$.

Now we are going to estimate $I_{2}$. The Holder inequality gives

$$
\begin{aligned}
& \left|\int_{\mathbb{R}^{n} \backslash \cup B_{j}} E \tilde{Z}_{n+1}\right| \\
& \leq C \\
& \quad \times(1+|y|)^{n+2 s-\frac{2 n}{q}} E \|_{L^{q}\left(\mathbb{R}^{n} \backslash \cup B_{j}\right)} \\
& \quad \times\left\|(1+|y|)^{-n-2 s+\frac{2 n}{q}} \mu^{\frac{n-2 s}{2}} Z_{n+1}\left(y+\mu^{-1}\left(\xi_{j}-\xi_{1}\right)\right)\right\|_{L^{q /(q-1)}\left(\mathbb{R}^{n} \backslash \cup B_{j}\right)} .
\end{aligned}
$$

A direct computation gives that

$$
\left\|(1+|y|)^{-n-2 s+\frac{2 n}{q}} \mu^{\frac{n-2 s}{2}} Z_{n+1}\left(y+\mu^{-1}\left(\xi_{j}-\xi_{1}\right)\right)\right\|_{L^{q /(q-1)}\left(\mathbb{R}^{n} \backslash \cup B_{j}\right)} \leq C k^{-n \frac{q-1}{q}}
$$

for some constant $C>0$. Thus we conclude that

$$
\left|I_{2}\right| \leq C k^{1-n}
$$

since we have already proved — see (13) — that

$$
\left\|(1+|y|)^{n+2 s-\frac{2 n}{q}} E\right\|_{L^{q}\left(\mathbb{R}^{n} \backslash \cup B_{j}\right)} \leq C k^{1-\frac{n}{q}} .
$$


Let $j \neq 1$ be fixed and $\tilde{E}_{j}(y)=\mu^{\frac{n+2 s}{2}} E\left(\xi_{j}+\mu y\right)$. After the change of variable $x=\mu y+\xi_{j}$, we obtain

$$
\begin{aligned}
\left|\int_{B_{j}} E \tilde{Z}_{n+1}\right|= & \left|\mu^{\frac{n-2 s}{2}} \int_{B\left(0, \frac{\eta}{\mu k}\right)} \tilde{E}_{j} \tilde{Z}_{n+1}\left(\mu y+\xi_{j}\right)\right| \\
\leq & C \mu^{\frac{n-2 s}{2}}\left\|(1+|y|)^{n+2 s-\frac{2 n}{q}} \tilde{E}_{j}\right\|_{L^{q}\left(B\left(0, \frac{\eta}{\mu k}\right)\right)} \\
& \times\left\|(1+|y|)^{-n-2 s+\frac{2 n}{q}} \mu^{\frac{n-2 s}{2}} Z_{n+1}\left(y+\mu^{-1}\left(\xi_{j}-\xi_{1}\right)\right)\right\|_{L^{q /(q-1)}\left(B\left(0, \frac{\eta}{\mu k}\right)\right)} .
\end{aligned}
$$

We have

$$
\begin{aligned}
\|(1+|y|)^{-n-2 s+\frac{2 n}{q}} \mu^{\frac{n-2 s}{2}} Z_{n+1}\left(y+\mu^{-1}\right. & \left.\left(\xi_{j}-\xi_{1}\right)\right) \|_{L^{q /(q-1)}\left(B\left(0, \frac{\eta}{\mu k}\right)\right)} \\
& \leq C \frac{\mu^{\frac{n-2 s}{2}}}{\left|\xi_{j}-\xi_{1}\right|^{n-2 s}}\left(\int_{1}^{\frac{\eta}{\mu k}} \frac{t^{n-1}}{t^{\left(n+2 s-\frac{2 n}{q}\right) \frac{q}{q-1}}} d t\right)^{\frac{q-1}{q}} \\
& \leq C \frac{\mu^{\frac{n-2 s}{2}}}{\left|\xi_{j}-\xi_{1}\right|^{n-2 s}}(\mu k)^{2 s-\frac{n}{q}}
\end{aligned}
$$

and

$$
\left\|(1+|y|)^{n+2 s-\frac{2 n}{q}} \tilde{E}_{j}\right\|_{L^{q}\left(B\left(0, \frac{\eta}{\mu k}\right)\right)} \leq(\mu k)^{\frac{n}{q}}\left(1+k^{-(n+2 s)} \mu^{-\frac{n-2 s}{2}}\right) .
$$

Hence,

$$
\begin{aligned}
\left|I_{3}\right| & =\left|\sum_{j \neq 1} \int_{B_{j}} E \tilde{Z}_{n+1}\right| \\
& \leq \mu^{\frac{n-2 s}{2}}(\mu k)^{\frac{n}{q}}\left(1+k^{-(n+2 s)} \mu^{-\frac{n-2 s}{2}}\right) \sum_{j=1}^{k} \frac{\mu^{\frac{n-2 s}{2}}}{\left|\xi_{j}-\xi_{1}\right|^{n-2 s}}(\mu k)^{2 s-\frac{n}{q}} \\
& \leq C \mu^{\frac{n-2 s}{2}} k^{-2 s}
\end{aligned}
$$

Finally, we conclude that

$$
\int_{\mathbb{R}^{n}} E \tilde{Z}_{n+1}=A \delta k^{-(n-2 s)}\left(-2^{\frac{n-2 s}{2}}\left(\sum_{j=1}^{\infty} \frac{1}{j^{n-2 s}}\right) \delta+1\right)+k^{1-n} \Theta_{k}(\delta),
$$

where $\Theta_{k}(\delta)$ is a smooth function of $\delta$, which is uniformly bounded as $k \rightarrow \infty$.

In order to complete the proof of (53), we first estimate:

$$
\left|\int_{\mathbb{R}^{n}}\left(\zeta_{1}-1\right) E \tilde{Z}_{n+1}\right| \leq C\left|\int_{\left|y-\xi_{1}\right|>\frac{\eta}{k}} E \tilde{Z}_{n+1}\right| .
$$


Then we split the domain of integration:

$$
\int_{\left|y-\xi_{1}\right|>\frac{\eta}{k}} E \tilde{Z}_{n+1}=\int_{\bigcap_{j}\left|y-\xi_{j}\right|>\frac{\eta}{k}} E \tilde{Z}_{n+1}+\sum_{j=2}^{k} \int_{\left|y-\xi_{j}\right|<\frac{\eta}{k}} E \tilde{Z}_{n+1}
$$

In the exterior region, we already proved that

$$
\int_{\bigcap_{j}\left|y-\xi_{j}\right|>\frac{\eta}{k}} E \tilde{Z}_{n+1}=k^{1-n} \Theta_{k}(\delta),
$$

for some smooth function $\Theta_{k}$ of $\delta$, which is uniformly bounded as $k \rightarrow \infty$. On the another hand, to estimate

$$
\sum_{j=2}^{k} \int_{\left|y-\xi_{j}\right|<\frac{\eta}{k}} E \tilde{Z}_{n+1}
$$

we can argue like in the estimate of the term $I_{3}$ above, thus concluding that

$$
\left|\sum_{j=2}^{k} \int_{\left|y-\xi_{j}\right|<\frac{\eta}{k}} E \tilde{Z}_{n+1}\right| \leq C k^{-n}
$$

for some constant $C>0$.

Proof of (54). It is convenient to decompose

$$
\mathcal{N}\left(\phi_{1}\right)=\tilde{\mathcal{N}}\left(\phi_{1}\right)+N\left(\tilde{\phi}_{1}\right)
$$

where

$$
\begin{aligned}
\tilde{\mathcal{N}}\left(\phi_{1}\right)=p\left(\left|U_{*}\right|^{p-1} \zeta_{1}-U_{1}^{p-1}\right) \tilde{\phi}_{1}+p \zeta_{1}\left|U_{*}\right|^{p-1} \Psi\left(\phi_{1}\right) \\
+N\left(\tilde{\phi}_{1}+\sum_{j \neq 1} \tilde{\phi}_{j}+\Psi\left(\phi_{1}\right)\right)-N\left(\tilde{\phi}_{1}\right)
\end{aligned}
$$

and

$$
N\left(\tilde{\phi}_{1}\right)=\left|U_{*}+\tilde{\phi}_{1}\right|^{p-1}\left(U_{*}+\tilde{\phi}_{1}\right)-\left|U_{*}\right|^{p-1} U_{*}-p\left|U_{*}\right|^{p-1} \tilde{\phi}_{1}
$$

We have that

$$
I:=\int_{\mathbb{R}^{n}} \tilde{\mathcal{N}}\left(\phi_{1}\right) \tilde{Z}_{n+1}=\mu^{\frac{n+2}{2}} \int_{\mathbb{R}^{n}} \tilde{\mathcal{N}}\left(\phi_{1}\right)\left(\xi_{1}+\mu x\right) Z_{n+1}(x) d x
$$

so that, from the estimates found, we readily check

$$
|I| \leq C k^{2 s-n} k^{1-\frac{n}{q}} \int_{R^{n}} U^{p-1}\left|Z_{n+1}\right| .
$$

On the other hand, if we let

$$
I I:=\int_{R^{n}} N\left(\tilde{\phi}_{1}\right) \tilde{Z}_{n+1},
$$


we find that

$$
|I I| \leq\left\|\phi_{1}\right\|_{*} \int_{R^{n}} U^{p-1}\left|\phi_{1}\right|\left|Z_{n+1}\right| .
$$

Now, we notice that from (23), we can write

$$
L_{0}\left(\phi_{1}\right)+a \phi_{1}=g+\sum_{l} c_{l} U^{p-1} Z_{l}, \quad \text { where } a=\mu^{\frac{n+2 s}{2}} \gamma N\left(\tilde{\phi}_{1}\right)\left(\xi_{1}+\mu y\right)
$$

so that

$$
|a| \leq C U^{p-1}\left\|\phi_{1}\right\|_{*} \quad \text { and } \quad|g| \leq C \mu^{\frac{n-2 s}{2}}(1+|y|)^{-4 s} .
$$

Thus, applying Lemma 3.3 with $v=4 s$, we find

$$
\left|\phi_{1}\right| \leq C \mu^{\frac{n-2 s}{2}}(1+|y|)^{-2 s}
$$

and we conclude that

$$
|I I| \leq C\left\|\phi_{1}\right\|_{*} \mu^{\frac{n-2 s}{2}} \leq C k^{2 s-n-\frac{n}{q}} .
$$

Combining this with (56), we find

$$
\left|\int_{R^{n}} \mathcal{N}\left(\phi_{1}\right) \tilde{Z}_{n+1}\right| \leq C k^{2 s-n} k^{1-\frac{n}{q}} .
$$

\section{Acknowledgments}

Musso is partially supported by Fondecyt Grant 1120151 and Millennium Nucleus Center for Analysis of PDE, NC130017. Garrido is partially supported by Mecesup Grant 0711 and VRI Scholarship.

\section{References}

[Caffarelli and Silvestre 2007] L. Caffarelli and L. Silvestre, "An extension problem related to the fractional Laplacian”, Comm. Partial Differential Equations 32 (2007), 1245-1260. MR 2009k:35096 Zbl 1143.26002

[Carlen and Loss 1990] E. A. Carlen and M. Loss, "Extremals of functionals with competing symmetries”, J. Funct. Anal. 88:2 (1990), 437-456. MR 91f:42021 Zbl 0705.46016

[Chang and González 2011] S.-Y. A. Chang and M. d. M. González, "Fractional Laplacian in conformal geometry", Adv. Math. 226:2 (2011), 1410-1432. MR 2012k:58057 Zbl 1214.26005

[Chen et al. 2006] W. Chen, C. Li, and B. Ou, "Classification of solutions for an integral equation", Comm. Pure Appl. Math. 59:3 (2006), 330-343. MR 2006m:45007a Zbl 1093.45001

[Dávila et al. 2013] J. Dávila, M. del Pino, and Y. Sire, "Nondegeneracy of the bubble in the critical case for nonlocal equations", Proc. Amer. Math. Soc. 141:11 (2013), 3865-3870. MR 3091775 Zbl 1275.35028

[Di Nezza et al. 2012] E. Di Nezza, G. Palatucci, and E. Valdinoci, "Hitchhiker's guide to the fractional Sobolev spaces", Bull. Sci. Math. 136:5 (2012), 521-573. MR 2944369 Zbl 1252.46023

[Fang 2014] F. Fang, "Infinitely many non-radial sign-changing solutions for a fractional Laplacian equation with critical nonlinearity”, preprint, 2014. arXiv 1408.3187 
[Frank and Lieb 2010] R. L. Frank and E. H. Lieb, "Inversion positivity and the sharp HardyLittlewood-Sobolev inequality", Calc. Var. Partial Differential Equations 39:1-2 (2010), 85-99. MR 2012a:26027 Zbl 1204.39024

[Frank and Lieb 2012] R. L. Frank and E. H. Lieb, "A new, rearrangement-free proof of the sharp Hardy-Littlewood-Sobolev inequality”, pp. 55-67 in Spectral theory, function spaces and inequalities, edited by B. M. Brown et al., Operator Theory: Advances and Applications 219, Birkhäuser, Basel, 2012. MR 2848628 Zbl 1297.39023

[González and Qing 2013] M. d. M. González and J. Qing, "Fractional conformal Laplacians and fractional Yamabe problems", Anal. PDE 6:7 (2013), 1535-1576. MR 3148060 Zbl 1287.35039

[Li 2004] Y. Y. Li, "Remark on some conformally invariant integral equations: the method of moving spheres”, J. Eur. Math. Soc. 6:2 (2004), 153-180. MR 2005e:45007 Zbl 1075.45006

[Li and Zhu 1995] Y. Y. Li and M. Zhu, "Uniqueness theorems through the method of moving spheres", Duke Math. J. 80:2 (1995), 383-417. MR 96k:35061 Zbl 0846.35050

[Lieb 1983] E. H. Lieb, "Sharp constants in the Hardy-Littlewood-Sobolev and related inequalities", Ann. of Math. (2) 118:2 (1983), 349-374. MR 86i:42010 Zbl 0527.42011

[del Pino et al. 2011] M. del Pino, M. Musso, F. Pacard, and A. Pistoia, "Large energy entire solutions for the Yamabe equation", J. Differential Equations 251:9 (2011), 2568-2597. MR 2012k:35176 Zbl 1233.35008

[del Pino et al. 2013] M. del Pino, M. Musso, F. Pacard, and A. Pistoia, "Torus action on $S^{n}$ and sign-changing solutions for conformally invariant equations", Ann. Sc. Norm. Super. Pisa Cl. Sci. (5) 12:1 (2013), 209-237. MR 3088442 Zbl 1267.53040

Received January 5, 2015.

DANILO GARRIDO

DePartamento de Matemática

Pontificia Universidad CATOlica de CHILE

AVDA. ViCUÑA MACKENNA 4860

$\mathrm{X}$ MACUL

CHILE

dggarrido@mat.puc.cl

MONICA MuSSO

Departmento de MATEMÁtica

Pontifica Universidad Catolica de Chile

AVDA. VICUÑA MACKENNA

4860 MACUL

CHILE

mmusso@mat.puc.cl 


\title{
PACIFIC JOURNAL OF MATHEMATICS
}

Founded in 1951 by E. F. Beckenbach (1906-1982) and F. Wolf (1904-1989)

$$
\text { msp.org/pjm }
$$

\section{EDITORS}

\author{
Don Blasius (Managing Editor) \\ Department of Mathematics \\ University of California \\ Los Angeles, CA 90095-1555 \\ blasius@math.ucla.edu
}

\author{
Paul Balmer \\ Department of Mathematics \\ University of California \\ Los Angeles, CA 90095-1555 \\ balmer@math.ucla.edu \\ Robert Finn \\ Department of Mathematics \\ Stanford University \\ Stanford, CA 94305-2125 \\ finn@math.stanford.edu \\ Sorin Popa \\ Department of Mathematics \\ University of California \\ Los Angeles, CA 90095-1555 \\ popa@math.ucla.edu
}

\author{
Vyjayanthi Chari \\ Department of Mathematics \\ University of California \\ Riverside, CA 92521-0135 \\ chari@math.ucr.edu \\ Kefeng Liu \\ Department of Mathematics \\ University of California \\ Los Angeles, CA 90095-1555 \\ liu@math.ucla.edu \\ Igor Pak \\ Department of Mathematics \\ University of California \\ Los Angeles, CA 90095-1555 \\ pak.pjm@gmail.com \\ Paul Yang \\ Department of Mathematics \\ Princeton University \\ Princeton NJ 08544-1000 \\ yang@math.princeton.edu
}

\section{PRODUCTION}

Silvio Levy, Scientific Editor, production@msp.org

\section{SUPPORTING INSTITUTIONS}

ACADEMIA SINICA, TAIPEI

CALIFORNIA INST. OF TECHNOLOGY

STANFORD UNIVERSITY

UNIV. OF BRITISH COLUMBIA

UNIV. OF CALIFORNIA, BERKELEY

UNIV. OF CALIFORNIA, DAVIS

UNIV. OF CALIFORNIA, LOS ANGELES

UNIV. OF CALIFORNIA, RIVERSIDE

UNIV. OF CALIFORNIA, SAN DIEGO

UNIV. OF CALIF., SANTA BARBARA
KEIO UNIVERSITY

MATH. SCIENCES RESEARCH INSTITUTE

NEW MEXICO STATE UNIV.

OREGON STATE UNIV.
Daryl Cooper

Department of Mathematics

University of California

Santa Barbara, CA 93106-3080 cooper@math.ucsb.edu

Jiang-Hua Lu

Department of Mathematics

The University of Hong Kong

Pokfulam Rd., Hong Kong

jhlu@maths.hku.hk

$$
\text { Jie Qing }
$$

Department of Mathematics

University of California

Santa Cruz, CA 95064

qing@cats.ucsc.edu

\author{
UNIV. OF CALIF., SANTA CRUZ \\ UNIV. OF MONTANA \\ UNIV. OF OREGON \\ UNIV. OF SOUTHERN CALIFORNIA \\ UNIV. OF UTAH \\ UNIV. OF WASHINGTON \\ WASHINGTON STATE UNIVERSITY
}

These supporting institutions contribute to the cost of publication of this Journal, but they are not owners or publishers and have no responsibility for its contents or policies.

See inside back cover or msp.org/pjm for submission instructions.

The subscription price for 2016 is US \$/year for the electronic version, and \$/year for print and electronic.

Subscriptions, requests for back issues and changes of subscriber address should be sent to Pacific Journal of Mathematics, P.O. Box 4163, Berkeley, CA 94704-0163, U.S.A. The Pacific Journal of Mathematics is indexed by Mathematical Reviews, Zentralblatt MATH, PASCAL CNRS Index, Referativnyi Zhurnal, Current Mathematical Publications and Web of Knowledge (Science Citation Index).

The Pacific Journal of Mathematics (ISSN 0030-8730) at the University of California, c/o Department of Mathematics, 798 Evans Hall \#3840, Berkeley, CA 94720-3840, is published twelve times a year. Periodical rate postage paid at Berkeley, CA 94704, and additional mailing offices. POSTMASTER: send address changes to Pacific Journal of Mathematics, P.O. Box 4163, Berkeley, CA 94704-0163.

PJM peer review and production are managed by EditFLOW ${ }^{\circledR}$ from Mathematical Sciences Publishers.

PUBLISHED BY

\section{I. mathematical sciences publishers}

nonprofit scientific publishing

http://msp.org/

(C) 2016 Mathematical Sciences Publishers 


\section{PACIFIC JOURNAL OF MATHEMATICS}

Volume $283 \quad$ No. $1 \quad$ July 2016

A New family of simple $\mathfrak{g l}_{2 n}(\mathbb{C})$-modules

JONATHAN NILSSON

Derived categories of representations of small categories over commutative noetherian rings

BENJAMIN ANTIEAU and GREg STEVENSON

Vector bundles over a real elliptic curve

INDRANIL BISWAS and FLORENT SCHAFFHAUSER

$\mathrm{Q}(\mathrm{N})$-graded Lie superalgebras arising from fermionic-bosonic representations

JIN CHENG

Conjugacy and element-conjugacy of homomorphisms of compact Lie groups

YiNGJUE FANG, GANG HAN and BINYONG SUN

Entire sign-changing solutions with finite energy to the fractional Yamabe equation

DANILO GARRIDO and MoniCa Musso

Calculation of local formal Mellin transforms

ADAM GRAHAM-SQUIRE

The untwisting number of a knot

KENAN INCE

A Plancherel formula for $L^{2}(G / H)$ for almost symmetric subgroups

Bent Ørsted and Birgit SPEH

Multiplicative reduction and the cyclotomic main conjecture for $\mathrm{GL}_{2}$

CHRISTOPHER SKINNER

Commensurators of solvable $S$-arithmetic groups

\section{DANIEL STUDENMUND}

Gerstenhaber brackets on Hochschild cohomology of quantum symmetric algebras and their group extensions

SARAH WITHERSPOON and GUODONG ZHOU 